\title{
On Investor Preferences and Mutual Fund Separation
}

\author{
Philip Dybvig* $\quad$ Fang $\mathrm{Liu}^{\dagger}$
}

July 2016

\begin{abstract}
We extend Cass and Stiglitz's analysis of preference-based mutual fund separation. We provide a complete characterization of the general $K$-fund separation. We show that some instances of high-degree separation can be constructed by adding inverse marginal utility functions exhibiting lower degrees of separation. We also show that there is money separation (in which we can choose the riskless asset as one of the funds) if and only if there is a fund (which may or may not be the riskless asset) with a constant allocation as wealth changes. In general, we do not know how to write the separating utility functions in closed form, but we can do so in the special case of SAHARA utility defined by Chen et al. and for a new class of GOBI preferences introduced here.
\end{abstract}

\section{Introduction}

Mutual fund separation is an important concept in portfolio selection. It means that all investors' optimal portfolio choices can be constructed as the linear combination of a set of mutual funds regardless of the initial wealth level, where a mutual fund can be any portfolio of tradable assets in the market. In other words, under mutual fund separation investors should be able to achieve the same level of utility from the individual assets as if they were only offered a set of mutual funds. The term "separation" comes from the fact that every investor can "separate" his portfolio choice into two steps. First, the investor chooses a small set of funds that spans optimal portfolios of all wealth levels. Second, the investor determines the optimal mixture of the separating funds based on his current wealth level.

The first results of mutual fund separation are developed under the mean-variance framework. Tobin (1958) finds that when investors only care about the mean and variance of the return distribution and in the presence of a riskless asset, optimal consumptions can be spanned by a risky portfolio and the riskless asset. He also shows

\footnotetext{
*Olin Business School, Washington University in St. Louis. E-mail: dybvig@wustl.edu.

${ }^{\dagger}$ School of Hotel Administration, Cornell University. E-mail: fang.liu@cornell.edu.
} 
that the mean-variance assumption is consistent with von Neumann-Morgenstern preferences with quadratic utility functions. Black (1972) proves that under meanvariance preferences, even in the absence of the riskless asset, similar two-fund separation results still hold with both separating funds being risky portfolios.

When agents do not have mean-variance preferences, some alternative assumption is needed to support mutual fund separation. Such conditions can be roughly classified into two types: those in terms of investor preferences and those in terms of the distributions of asset returns. Among research examining preference-based separation, Pye (1967) and Hakansson (1969) find that the hyperbolic absolute risk aversion (HARA) class exhibits two-fund separation with one of the separating funds being the riskless asset. Cass and Stiglitz (1970) further characterize the class of preferences that permits mutual fund separation, regardless of the distributions of asset returns. On the return distribution side, efforts have been made to delineate the class of stochastic processes that supports separation for all utility functions. Samuelson (1967) shows that if asset payoffs are independent and identically distributed, all risk-averse agents will choose the equally-weighted portfolio, and more generally Ross (1978) derives necessary and sufficient conditions on the stochastic structure of asset returns such that mutual fund separation can be sustained, independent of investor preferences.

In this paper, we extend Cass and Stiglitz's analysis of preference-based mutual fund separation. While Cass and Stiglitz mostly focus on one- and two-fund separation, we emphasize the general $K$-fund separation. ${ }^{1}$ We provide a complete characterization of the general $K$-fund separation in terms of the inverse marginal utility function. We show that high-degree separation can be constructed by adding low-degree separating preferences in the inverse marginal utility function. However, this method does not allow us to find all utility functions satisfying fund separation. We also study money separation in which we can choose the riskless asset as one of the separating funds. We show that money separation holds if and only if there is a fund (the riskless asset or a risky portfolio) whose optimal allocation is constant and does not depend on initial wealth. While it is generally hard, if not impossible, to

\footnotetext{
${ }^{1}$ Cass and Stiglitz (1970) do have a result on high-degree separation, their confusing Theorem 7.1, which is presented without proof. Unfortunately, this is a weak point in a great paper, and seems to be incorrect if only because the trigonometric terms are missing. Given the Remark after the statement of the theorem and the discussion in footnote 1 in Appendix II, it seems to be assumed that the trigonometric terms are ruled out in general by concavity, like they are for one- and two-fund separation.
} 
write the utility function of a separating preference in closed form, we show that this can be achieved for two special classes of preferences, both of which exhibit three-fund separation. We also make a minor contribution to the theory of one- and two-fund separation. Mirror CRRA preferences have a functional form like constant relative risk aversion (CRRA) preferences, but are defined on negative wealth. We also consider two-fund separating preferences constructed from mirror CRRA preferences, perhaps in combination with CRRA preferences.

The study of mutual fund separation has important implications. If we have reasons to believe that $K$-fund separation holds, where $K$ is a relatively small number, then a money manager could set up a relatively small set of commingled portfolios, active or passive according to the manager's style, to serve all clients at all levels of wealth. The optimal combination of these commingled portfolios would deliver the same payoff and therefore the same level of utility as individualized portfolios constructed optimally from the individual assets for each client. In other words, we could set up the $K$ separating funds as index funds in a fund family, and these funds are all that an investor would ever need to trade.

It is also useful to study mutual fund separation because it helps to motivate new tractable functional forms of utility functions. In many important finance problems such as portfolio selection and asset pricing, fund separation often simplifies the analysis. While most existing work focuses on one- and two-fund separation, we show that higher degrees of separation are rich and interesting but still tractable.

We consider a one-period setting: investors with von Neumann-Morgenstern preferences invest at the beginning of the period and consume at the end. Given complete markets with a unique stochastic discount factor, the optimal consumption portfolio is determined by the first order condition, which says that the marginal utility at optimum is proportional to the stochastic discount factor. Strict concavity then enables us to invert the marginal utility function and express the optimal portfolio as the inverse marginal utility evaluated at the stochastic discount factor multiplied by the shadow price which depends on the initial wealth level. This approach allows us to prove a general characterization of preference-based $K$-fund separation in terms of the inverse marginal utility, which includes $K$-fund separating preferences that can be generated by combining one-fund separating preferences, as well as some that cannot. Various examples allow us to explore how this works.

Following Cass and Stiglitz (1970), we say there is money separation if there is 
separation and one of the funds can be chosen to be the riskless asset. We show that money separation holds if and only if we can choose a separating fund whose optimal investment weight is constant and independent of the initial wealth level. Interestingly, the constant weight can be assigned to either the riskless asset (e.g., quadratic utility) or a risky fund (e.g., constant absolute risk aversion (CARA) utility). In addition, we also show that money separation is closely related to shifts in the utility function. A non-money separating utility function can be converted into a money separating utility function by adding a constant to the inverse marginal utility, or equivalently through a translation of consumption, as when we go from CRRA utility to HARA utility with the same exponent.

The characterization of separating utility functions gives the inverse marginal utility in closed form. From this, we can derive the utility function itself by first inverting the inverse marginal utility to obtain the marginal utility, and then integrating the marginal utility to obtain the utility function. This is easy numerically, but unfortunately does not yield a closed-form expression except in a few special cases. Aside from the one- and two-fund separating cases already in the literature, we discuss two cases with closed-form expressions for the utility function. One is the symmetric asymptotic hyperbolic absolute risk aversion (SAHARA) preferences proposed by Chen, Pelsser and Vellekoop (2011), and another is the GOBI preferences to be introduced in this paper. Both classes exhibit three-fund separation, and they have not only a simple form in the inverse marginal utility, but a closed-form expression in the utility function itself.

Concavity imposes additional constraints on our characterization of separation. A separating utility function is strictly concave if and only if the inverse marginal utility is monotonically decreasing everywhere. ${ }^{2}$ It is hard to derive necessary and sufficient conditions for strict concavity in terms of the parameter values, something similar to finding conditions for a polynomial to be positive everywhere. Nonetheless, we analyze specific examples and we give sufficient conditions to be inconsistent with any strictly concave utility function.

Finally, we extend our analyses from the von Neumann-Morgenstern expected utility to a broader set of Machina preferences. According to Machina (1982), when the utility function is smooth enough, these preferences can be locally modeled as

\footnotetext{
${ }^{2}$ This is not true for all potential utility functions, for example one with a kink, but separating utility functions are in a restricted class.
} 
expected utility. We show that if the local utility function satisfies fund separation everywhere with the same set of separating funds, this is sufficient but not necessary for global fund separation. We illustrate this using examples.

In this paper, we follow Cass and Stiglitz (1970) and consider complete markets only. In general, separation need not hold under conditions of the theorems when markets are incomplete, with some known exceptions in the literature. Hakansson (1969) shows that one-fund separation still holds for logarithm and power utility even if markets are incomplete, and in the presence of a riskless asset, the HARA class exhibits two-fund separation even if markets are incomplete. Black (1972) shows that quadratic utility satisfies two-fund separation under incomplete markets even without a riskless asset. Beyond this short list of known examples, the sufficient conditions provided in this paper are no longer sufficient if markets are incomplete, and we can construct numerical examples where two-fund separation fails when markets are incomplete. ${ }^{3}$

The rest of the paper proceeds as follows. Section 2 defines mutual fund separation and characterizes the class of separating preferences in terms of the inverse marginal utility function. We also demonstrate how low-degree separation can be used to construct high-degree separating preferences. Section 3 studies money separation. Section 4 derives the utility functions for the SAHARA and GOBI preferences, both of which exhibit three-fund separation. Section 5 examines conditions for strict concavity and discusses how they can be used to refine the separating class. Section 6 discusses sufficient conditions for fund separation for Machina preferences. Section 7 concludes the paper. Some intuitive proofs are included in the main text, while other proofs are relegated to the Appendix.

\section{Mutual Fund Separation}

In this section, we study necessary and sufficient conditions for preference-based $K$ fund separation.

\section{$2.1 \quad$ Setup}

Following Cass and Stiglitz (1970), we consider a one-period model, in which investors invest at the beginning of the period and consume at the end. Assume that markets

\footnotetext{
${ }^{3}$ As in Dybvig and Ross (1982), the minimal example has three assets and four states, for similar reasons.
} 
are complete and there exists a unique stochastic discount factor $\rho>0$ that takes all positive values with $\mathbf{E}(\rho)<\infty$. We also use $\rho$ to represent realizations of the random stochastic discount factor. Assume that each investor has a von NeumannMorgenstern utility function $u(\cdot)$ defined on any open interval $D \subset \mathbb{R}$, which is twice differentiable with $u^{\prime}>0$ and $u^{\prime \prime}<0$. We allow for both positive and negative consumption levels. ${ }^{4}$ We denote the set of utility functions of all investors by $\mathcal{U}$. Then, an investor with utility function $u \in \mathcal{U}$ and initial wealth $w_{0} \in \mathbb{R}$ solves the following utility maximization problem.

Problem 1 Choose consumption $x$ to

$$
\max _{x} \mathbf{E}[u(x)]
$$

subject to the budget constraint

$$
\mathbf{E}(x \rho) \leq w_{0}
$$

We denote the set of solutions to Problem 1 by $\mathcal{S}\left(u, w_{0}\right)$. By strict concavity of $u, \mathcal{S}\left(u, w_{0}\right)$ is either an empty set or a singleton. Assume that for all utility functions under consideration, there exists an open interval for the initial wealth such that an optimum to Problem 1 exists, i.e., $\mathcal{S}\left(u, w_{0}\right) \neq \varnothing$. Now we define $K$-fund separation if there are no fewer than $K$ mutual funds whose random payoffs span the optimal consumptions of all investors whenever an optimum exists, regardless of the initial wealth level.

Definition 1 We have $K$-fund separation if $K$ is the smallest positive integer such that there exist $K$ mutual funds $\left\{f_{k}(\rho)\right\}_{k=1, \ldots, K}$, which satisfy that for all $u \in \mathcal{U}$ and $w_{0} \in \mathbb{R}$, if $\mathcal{S}\left(u, w_{0}\right) \neq \varnothing$, then we can find $\left\{\alpha_{k}\left(u, w_{0}\right)\right\}_{k=1, \ldots, K}$ such that $\sum_{k=1}^{K} \alpha_{k}\left(u, w_{0}\right) f_{k}(\rho) \in \mathcal{S}\left(u, w_{0}\right)$.

Several comments are worth pointing out. First, the optimal consumptions and the separating funds are both identified in terms of payoff, whereas the associated portfolio compositions may not be uniquely determined in the presence of redundant assets. Second, whenever $K$-fund separation holds for $K \geq 2$, the set of separating funds is not unique. Indeed, having one set of separating funds, we can easily

\footnotetext{
${ }^{4}$ While negative consumption may seem absurd on its face, what we call consumption might be the net trade or it can be justified by a promise to do work to cover any negative amount. Also, even if consumption is not literally negative, it can be a useful modelling device.
} 
construct another by taking linear combinations of the original set of funds, and the resulting investment weights are also linear combinations of the original weights. Finally, while $K$-fund separation is defined for a set of utility functions, we are often interested in $K$-fund separation for a single utility function as a special case, which is obtained when $\mathcal{U}$ contains one utility function only.

One special form of mutual fund separation obtains when we can choose the riskless asset as one of the separating funds. We follow Cass and Stiglitz (1970) and refer to this special case as money separation. In other words, money separation holds as long as the riskless asset is in the linear span of the separating funds. Formally, we have the following definition.

Definition 2 We have $K$-fund money separation if $K$-fund separation holds and we can choose $f_{1}(\rho)=1 .^{5}$

To characterize utility functions exhibiting mutual fund separation, we solve Problem 1. Suppose a solution exists, then the first order condition implies that the optimal consumption portfolio is given by

$$
x^{*}=I(\lambda \rho),
$$

where $I=\left(u^{\prime}\right)^{-1}$ is the inverse marginal utility function, and $\lambda>0$ is the shadow price whose value depends on the initial wealth level $w_{0}$. Since $u^{\prime \prime}<0$, it is apparent that $I$ exists and is unique. Preferences are unchanged by an increasing affine transformation of the utility function $u$ or equivalently by the rescaling of the argument of the inverse marginal utility function $I$. We will not distinguish different utility functions or inverse marginal utility functions that represent the same preferences.

If the utility function $u$ satisfies $K$-fund separation, then the optimal consumption (1) can be written as the weighted sum of $K$ mutual funds, with the associated weights depending on the initial wealth $w_{0}$ and thus on the shadow price $\lambda$, i.e.,

$$
I(\lambda \rho)=\sum_{k=1}^{K} \alpha_{k}(\lambda) f_{k}(\rho) .
$$

Notice that to ensure non-degeneracy, we must have that the separating funds $f_{k}(\rho)$ are linearly independent, and that the associated investment weights $\alpha_{k}(\lambda)$ are also

\footnotetext{
${ }^{5}$ The payoff to the riskless asset can take any constant value. Without loss of generality, we normalize it to be equal to 1 .
} 
linearly independent. Otherwise, the degree of separation can always be reduced by combining two or more funds to form a larger separating fund. In addition, for tractability, we only consider cases in which $\alpha_{k}(\lambda)$ are analytic functions. ${ }^{6}$

\section{$2.2 \quad$ Some Examples}

Before we formally characterize the set of separating preferences, let us first look at a few examples. Some of the following examples involve very well-known preferences, while others are less so. One might wonder how we come up with the more obscure examples. In fact, these examples are motivated by the general characterization of separating preferences to be introduced in the next section.

Example 1 (CRRA utility) Consider the CRRA utility function

$$
u(x)= \begin{cases}\frac{x^{1-R}}{1-R}, & R>0 \text { and } R \neq 1 \\ \log x, & R=1\end{cases}
$$

defined on all $x \in(0,+\infty)$, where $R$ is the coefficient of relative risk aversion. The inverse marginal utility function is given by

$$
I(\xi)=\xi^{-\frac{1}{R}}
$$

Since

$$
I(\lambda \rho)=(\lambda \rho)^{-\frac{1}{R}}=\lambda^{-\frac{1}{R}} \rho^{-\frac{1}{R}},
$$

we know from (2) that the CRRA utility function exhibits one-fund separation with separating fund

$$
f(\rho)=\rho^{-\frac{1}{R}}
$$

and corresponding investment weight

$$
\alpha(\lambda)=\lambda^{-\frac{1}{R}}
$$

Hence, an investor with CRRA utility would always find it optimal to invest his entire wealth into a single mutual fund $\rho^{-\frac{1}{R}}$, regardless of the initial wealth level.

Example 2 (Quadratic utility) Consider the quadratic utility function

$$
u(x)=-x^{2}+2 b x,
$$

\footnotetext{
${ }^{6} \mathrm{~A}$ function $f(\cdot)$ is said to be analytic if for any $x_{0}$ in the interior of its domain, the Taylor series of $f$ around $x_{0}$ converges to $f\left(x_{0}\right)$ on an interval of positive radius centered at $x_{0}$.
} 
where $x<b$. The inverse marginal utility function is given by

$$
I(\xi)=b-\frac{1}{2} \xi
$$

Since

$$
I(\lambda \rho)=b-\frac{1}{2} \lambda \rho
$$

the quadratic utility function exhibits two-fund money separation with separating funds

$$
\begin{aligned}
& f_{1}(\rho)=1, \\
& f_{2}(\rho)=\rho,
\end{aligned}
$$

and corresponding investment weights

$$
\begin{aligned}
& \alpha_{1}(\lambda)=b, \\
& \alpha_{2}(\lambda)=-\frac{1}{2} \lambda .
\end{aligned}
$$

Hence, an investor with quadratic utility would optimally invest a fixed amount $b$ into the riskless asset and take a wealth-dependent short position in the risky portfolio $\rho$.

Example 3 (SAHARA utility) The SAHARA preferences are introduced in Chen, Pelsser and Vellekoop (2011). They show that for a SAHARA utility function with scale parameter $b>0$ and risk aversion parameter $a>0$, the inverse marginal utility is given by

$$
I(\xi)=\frac{1}{2}\left(\xi^{-\frac{1}{a}}-b^{2} \xi^{\frac{1}{a}}\right)
$$

Since

$$
I(\lambda \rho)=\frac{1}{2}\left((\lambda \rho)^{-\frac{1}{a}}-b^{2}(\lambda \rho)^{\frac{1}{a}}\right)=\frac{1}{2} \lambda^{-\frac{1}{a}} \rho^{-\frac{1}{a}}-\frac{1}{2} b^{2} \lambda^{\frac{1}{a}} \rho^{\frac{1}{a}},
$$

the SAHARA utility function exhibits two-fund separation with separating funds

$$
\begin{aligned}
& f_{1}(\rho)=\rho^{-\frac{1}{a}}, \\
& f_{2}(\rho)=\rho^{\frac{1}{a}},
\end{aligned}
$$

and corresponding investment weights

$$
\begin{aligned}
& \alpha_{1}(\lambda)=\frac{1}{2} \lambda^{-\frac{1}{a}}, \\
& \alpha_{2}(\lambda)=-\frac{1}{2} b^{2} \lambda^{\frac{1}{a}} .
\end{aligned}
$$

Hence, an investor with SAHARA utility would always find it optimal to take a long position in fund $\rho^{-\frac{1}{a}}$ and a short position in fund $\rho^{\frac{1}{a}}$. 
A common feature of the above three examples is that their inverse marginal utility functions can all be viewed as linear combinations of power terms $\xi^{\gamma}$. In the CRRA case, there is only one power term $\xi^{-\frac{1}{R}}$; in the quadratic case, there are two power terms 1 and $\xi$ corresponding to $\gamma=0,1$; in the SAHARA case, there are again two power terms $\xi^{\frac{1}{a}}$ and $\xi^{-\frac{1}{a}}$. While one may suspect that the power terms are the only functional form permitted by separability, the following example demonstrates that the $\operatorname{logarithm}$ term $\log \xi$ can also show up.

Example 4 (CARA utility) Consider the CARA utility function

$$
u(x)=-e^{-A x},
$$

with the coefficient of absolute risk aversion $A>0$. The inverse marginal utility function is given by

$$
I(\xi)=\frac{1}{A}(\log A-\log \xi)
$$

Since

$$
I(\lambda \rho)=\frac{1}{A}(\log A-\log (\lambda \rho))=\frac{1}{A}(\log A-\log \lambda)-\frac{1}{A} \log \rho,
$$

the CARA utility function exhibits two-fund money separation with separating funds

$$
\begin{aligned}
& f_{1}(\rho)=1, \\
& f_{2}(\rho)=\log \rho,
\end{aligned}
$$

and corresponding investment weights

$$
\begin{aligned}
& \alpha_{1}(\lambda)=\frac{1}{A}(\log A-\log \lambda), \\
& \alpha_{2}(\lambda)=-\frac{1}{A} .
\end{aligned}
$$

Hence, an investor with CARA utility would always find it optimal to invest a wealthdependent amount into the riskless asset and take a constant short position in the risky portfolio $\log \rho$.

In all four examples above, whenever a power term $\xi^{\gamma}$ shows up in the inverse marginal utility function, $\gamma$ always takes real values. However, this does not have to be the case. When we have a pair of complex power values $\gamma \pm b i, \xi^{\gamma \pm b i}$ can be transformed into $\cos (b \log \xi) \xi^{\gamma}$ and $\sin (b \log \xi) \xi^{\gamma}$. The following example demonstrates that these terms can also appear in a separating preference. 
Example 5 Consider a utility function u, whose inverse marginal utility is given by

$$
I(\xi)=[\cos (\log \xi)+\sin (\log \xi)+2] \xi^{-2} .
$$

It can be verified that $I^{\prime}(\xi)<0$ (corresponding to concavity of utility) and

$$
\begin{aligned}
I(\lambda \rho)= & {[\cos (\log (\lambda \rho))+\sin (\log (\lambda \rho))+2](\lambda \rho)^{-2} } \\
= & {[\cos (\log \lambda)+\sin (\log \lambda)] \lambda^{-2} \cos (\log \rho) \rho^{-2} } \\
& +[\cos (\log \lambda)-\sin (\log \lambda)] \lambda^{-2} \sin (\log \rho) \rho^{-2}+2 \lambda^{-2} \rho^{-2} .
\end{aligned}
$$

Hence, we have three-fund separation with separating funds

$$
\begin{aligned}
& f_{1}(\rho)=\cos (\log \rho) \rho^{-2} \\
& f_{2}(\rho)=\sin (\log \rho) \rho^{-2} \\
& f_{3}(\rho)=\rho^{-2}
\end{aligned}
$$

and corresponding investment weights

$$
\begin{aligned}
& \alpha_{1}(\lambda)=[\cos (\log \lambda)+\sin (\log \lambda)] \lambda^{-2}, \\
& \alpha_{2}(\lambda)=[\cos (\log \lambda)-\sin (\log \lambda)] \lambda^{-2}, \\
& \alpha_{3}(\lambda)=2 \lambda^{-2} .
\end{aligned}
$$

\subsection{General Characterization of $K$-Fund Separation}

In this section, we provide a general characterization of preference-based $K$-fund separation. Our characterization is stated in terms of the inverse marginal utility function $I$. We show that the inverse marginal utility of a separating preference can only have the following terms: $C$ (constant), $\xi^{\gamma},(\log \xi)^{l}, \xi^{\gamma}(\log \xi)^{l}, \cos (b \log \xi), \sin (b \log \xi)$, $\xi^{\gamma} \cos (b \log \xi), \xi^{\gamma} \sin (b \log \xi),(\log \xi)^{l} \cos (b \log \xi),(\log \xi)^{l} \sin (b \log \xi), \xi^{\gamma}(\log \xi)^{l} \cos (b \log \xi)$, and $\xi^{\gamma}(\log \xi)^{l} \sin (b \log \xi)$. Indeed, we have already seen many of these terms in the examples above.

The following theorem provides the necessary and sufficient conditions for $K$-fund separation, where $K \geq 1$ can be any positive integer. This characterization is similar to Theorem 7.1 in Cass and Stiglitz (1970), although their result is stated without proof and seems to be incorrect if only because the trigonometric terms are missing. Their result also seems to contain terms that should not be there. However, the remark to the theorem describes an additional restriction which rules out at least some of the extra terms. 
Theorem 1 A utility function $u\left(\right.$ with $u^{\prime}>0$ and $\left.u^{\prime \prime}<0\right)$ exhibits $K$-fund separation if and only if the inverse marginal utility function $I=\left(u^{\prime}\right)^{-1}$ can be expressed as

$$
I(\xi)=\sum_{k=1}^{J} \xi^{\gamma_{k}} P_{k, 1}(\log \xi) \cos \left(b_{k} \log \xi\right)+\sum_{k=1}^{J} \xi^{\gamma_{k}} P_{k, 2}(\log \xi) \sin \left(b_{k} \log \xi\right),
$$

where

(1) The ordered pairs $\left(\gamma_{k}, b_{k}\right)$ are distinct for each $k$ with $b_{k} \geq 0$;

(2) For $i=1,2, P_{k, i}(\log \xi)$ is a polynomial function of $\log \xi$ of degree $d_{k, i} \geq 0$, i.e. $P_{k, i}(\log \xi)=\sum_{j=0}^{d_{k, i}} C_{k, i, j}(\log \xi)^{j}$, where the leading coefficient $C_{k, i, d_{k, i}} \neq 0$;

(3) If $b_{k}=0$ (the sin terms disappear, but the cos terms do not), then $d_{k, 2}=0$; and

(4) $\sum_{k=1}^{J}\left(d_{k}+1\right)\left(1+\mathbf{1}_{b_{k} \neq 0}\right)=K$, where $d_{k}=\max _{i=1,2}\left(d_{k, i}\right)$, and $\mathbf{1}_{b_{k} \neq 0}$ is an indicator function that takes a value of 1 when $b_{k} \neq 0$ and 0 otherwise.

The separating funds can be chosen as follows: $\forall k=1,2, \cdots, J$ and $\forall l=0,1, \cdots, d_{k}$,

$$
f_{k, l}(\rho)=\rho^{\gamma_{k}}(\log \rho)^{l}
$$

when $b_{k}=0$, and

$$
\begin{aligned}
& f_{k, 1, l}(\rho)=\rho^{\gamma_{k}} \cos \left(b_{k} \log \rho\right)(\log \rho)^{l}, \\
& f_{k, 2, l}(\rho)=\rho^{\gamma_{k}} \sin \left(b_{k} \log \rho\right)(\log \rho)^{l},
\end{aligned}
$$

when $b_{k} \neq 0$.

The associated investment weights are given by

$$
\alpha_{k, l}(\lambda)=\lambda^{\gamma_{k}} \sum_{j=l}^{d_{k, 1}} C_{k, 1, j}\left(\begin{array}{l}
j \\
l
\end{array}\right)(\log \lambda)^{j-l}
$$

when $b_{k}=0$, and

$$
\begin{aligned}
\alpha_{k, 1, l}(\lambda)= & \mathbf{1}_{l \leq d_{k, 1}} \lambda^{\gamma_{k}} \cos \left(b_{k} \log \lambda\right) \sum_{j=l}^{d_{k, 1}} C_{k, 1, j}\left(\begin{array}{c}
j \\
l
\end{array}\right)(\log \lambda)^{j-l} \\
& +\mathbf{1}_{l \leq d_{k, 2}} \lambda^{\gamma_{k}} \sin \left(b_{k} \log \lambda\right) \sum_{j=l}^{d_{k, 2}} C_{k, 2, j}\left(\begin{array}{c}
j \\
l
\end{array}\right)(\log \lambda)^{j-l}, \\
\alpha_{k, 2, l}(\lambda)= & \mathbf{1}_{l \leq d_{k, 2}} \lambda^{\gamma_{k}} \cos \left(b_{k} \log \lambda\right) \sum_{j=l}^{d_{k, 2}} C_{k, 2, j}\left(\begin{array}{c}
j \\
l
\end{array}\right)(\log \lambda)^{j-l} \\
& -\mathbf{1}_{l \leq d_{k, 1}} \lambda^{\gamma_{k}} \sin \left(b_{k} \log \lambda\right) \sum_{j=l}^{d_{k, 1}} C_{k, 1, j}\left(\begin{array}{c}
j \\
l
\end{array}\right)(\log \lambda)^{j-l},
\end{aligned}
$$


when $b_{k} \neq 0$.

Proof of Theorem 1 (sketch): Here is a sketch of the proof, which focuses on the necessity of (3). The complete formal proof is relegated to the Appendix.

Since the utility function $u$ exhibits $K$-fund separation, we must have that (2) holds whenever a solution to Problem 1 exists. Taking derivatives of (2) with respect to $\lambda$ yields

$$
\left(\begin{array}{c}
\rho I^{(1)}(\lambda \rho) \\
\vdots \\
\rho^{K} I^{(K)}(\lambda \rho)
\end{array}\right)=M_{0}(\lambda)\left(\begin{array}{c}
f_{1}(\rho) \\
\vdots \\
f_{K}(\rho)
\end{array}\right)
$$

where $I^{(k)}(\cdot)$ denotes the $k^{\text {th }}$ derivative of $I(\cdot)$, and $M_{0}(\lambda)$ is defined as

$$
M_{0}(\lambda)=\left(\begin{array}{ccc}
\alpha_{1}^{(1)}(\lambda) & \cdots & \alpha_{K}^{(1)}(\lambda) \\
\vdots & \ddots & \vdots \\
\alpha_{1}^{(K)}(\lambda) & \cdots & \alpha_{K}^{(K)}(\lambda)
\end{array}\right)
$$

Assume for now that $M_{0}(\lambda)$ is non-singular for some $\lambda$, i.e., $\exists \lambda$ such that $\left(M_{0}(\lambda)\right)^{-1}$ exists. We show in the appendix that a simple trick allows us to tackle the singularity case for which similar results obtain. When $M_{0}(\lambda)$ is not singular, we have

$$
\left(\begin{array}{c}
f_{1}(\rho) \\
\vdots \\
f_{K}(\rho)
\end{array}\right)=\left(M_{0}(\lambda)\right)^{-1}\left(\begin{array}{c}
\rho I^{(1)}(\lambda \rho) \\
\vdots \\
\rho^{K} I^{(K)}(\lambda \rho)
\end{array}\right) .
$$

Plugging (11) back into (2) gives

$$
I(\lambda \rho)=\left(\begin{array}{c}
\alpha_{1}(\lambda) \\
\vdots \\
\alpha_{K}(\lambda)
\end{array}\right)^{T}\left(M_{0}(\lambda)\right)^{-1}\left(\begin{array}{c}
\rho I^{(1)}(\lambda \rho) \\
\vdots \\
\rho^{K} I^{(K)}(\lambda \rho)
\end{array}\right) .
$$

Without loss of generality, assume that $\left(M_{0}(\lambda)\right)^{-1}$ exists when $\lambda=1$. Evaluating (12) at $\lambda=1$ and rearranging yield a differential equation of the form

$$
A_{K} I^{(K)}(\xi) \xi^{K}+\cdots+A_{1} I^{(1)}(\xi) \xi+I(\xi)=0,
$$

where $A_{1}, A_{2}, \cdots, A_{K}$ are constants. To ensure non-degenerate $K$-fund separation, we must have $A_{K} \neq 0$. Then, (13) is a $K^{\text {th }}$-order homogeneous Euler differential equation. 
To solve this differential equation, we conjecture $I(\xi)=\xi^{\delta}$ and plug this into (13). This gives us the following $K^{\text {th }}$-order polynomial equation

$$
A_{K} \delta(\delta-1) \cdots(\delta-K+1)+\cdots+A_{2} \delta(\delta-1)+A_{1} \delta+1=0
$$

with $K$ roots. Some of these $K$ roots may be repeated, thus reducing to $J \leq K$ distinct roots $\left\{\gamma_{k}+b_{k} i\right\}_{k=1}^{J}$, each of which can be either real $\left(b_{k}=0\right)$ or complex $\left(b_{k} \neq 0\right)$. If a real root $\gamma_{k}$ is repeated for $d_{k}+1$ times $\left(d_{k}=0\right.$ means that the root is not repeated), it gives rise to $d_{k}+1$ terms $\left\{\xi^{\gamma_{k}}(\log \xi)^{l}\right\}_{l=0}^{d_{k}}$ in $I(\xi)$, which can be combined as $\xi^{\gamma_{k}} P_{k, 1}(\log \xi)$. If a pair of complex roots $\gamma_{k} \pm b_{k} i$ is repeated for $d_{k}+1$ times, it then generates $\xi^{\gamma_{k}} P_{k, 1}(\log \xi) \cos \left(b_{k} \log \xi\right)$ and $\xi^{\gamma_{k}} P_{k, 2}(\log \xi) \sin \left(b_{k} \log \xi\right)$ in $I(\xi)$. To ensure that the total number of roots is equal to $K$, we must have

$$
K=\sum_{k=1}^{J}\left(d_{k}+1\right)\left(1+\mathbf{1}_{b_{k} \neq 0}\right) .
$$

Combining all the above terms, we have that $I(\xi)$ takes the form of $(3)$.

While (3) seems complicated, it is indeed a concise way to incorporate all possible terms in $I$ listed at the beginning of the section. The following table summarizes different possible terms in $I$ and the corresponding parameter values.

\begin{tabular}{lccc}
\hline Possible terms in $I$ & $\gamma_{k}$ & $b_{k}$ & $l$ \\
\hline$C$ (constant) & 0 & 0 & 0 \\
$\xi^{\gamma}$ & $\gamma$ & 0 & 0 \\
$(\log \xi)^{l}$ & 0 & 0 & $\geq 0$ \\
$\xi^{\gamma}(\log \xi)^{l}$ & $\gamma$ & 0 & $\geq 0$ \\
$\cos (b \log \xi), \sin (b \log \xi)$ & 0 & $b$ & 0 \\
$\xi^{\gamma} \cos (b \log \xi), \xi^{\gamma} \sin (b \log \xi)$ & $\gamma$ & $b$ & 0 \\
$(\log \xi)^{l} \cos (b \log \xi),(\log \xi)^{l} \sin (b \log \xi)$ & 0 & $b$ & $\geq 0$ \\
$\xi^{\gamma}(\log \xi)^{l} \cos (b \log \xi), \xi^{\gamma}(\log \xi)^{l} \sin (b \log \xi)$ & $\gamma$ & $b$ & $\geq 0$ \\
\hline
\end{tabular}

The characterization of $K$-fund separation for a class of preferences $\mathcal{U}$ follows almost immediately from Theorem 1 . The inverse marginal utility of each $u \in \mathcal{U}$ must be the sum of terms as in (3), and the leading coefficient on each of these terms must be non-zero for some utility function $\hat{u} \in \mathcal{U}$ to ensure non-degeneracy. Formally, we have the following corollary.

Corollary 1 A class of preferences $\mathcal{U}$ exhibits $K$-fund separation if and only if there exist $J$ distinct ordered pairs $\left\{\left(\gamma_{k}, b_{k}\right)\right\}_{k=1}^{J}$ with $b_{k} \geq 0$ and non-negative integers 
$\left\{D_{k}\right\}_{k=1}^{J}$ that satisfy $\sum_{k=1}^{J}\left(D_{k}+1\right)\left(1+\mathbf{1}_{b_{k} \neq 0}\right)=K$ such that $\forall u \in \mathcal{U}$, the inverse marginal utility function $I=\left(u^{\prime}\right)^{-1}$ can be expressed as (3), where

(1) For $i=1,2, P_{k, i}(\log (\xi))$ is a polynomial function of $\log (\xi)$ of degree $d_{k, i} \geq$ -1 . When $d_{k, i}=-1, P_{k, i}(\log (\xi))$ is an empty sum, which we take to be uniformly equal to zero;

(2) If $b_{k}=0$ (the sin terms disappear, but the cos terms do not), then $d_{k, 2}=-1$;

(3) $\forall k=1,2, \cdots, J, \max _{i=1,2} d_{k, i} \leq D_{k}$;

(4) $\forall k=1,2, \cdots, J, \exists \hat{u} \in \mathcal{U}$ such that $\max _{i=1,2} \hat{d}_{k, i}=D_{k}$.

Mutual fund separation for a class of preferences is very similar to that for a single utility function. Hence, we focus on fund separation for a single utility function in the analyses below.

\subsection{From Low-Degree to High-Degree Separation}

Theorem 1 provides a simple way of constructing higher-degree separating preferences from those with lower degrees. We now state it below.

Theorem 2 Consider $N$ utility functions $\left\{u_{n}\right\}_{n=1}^{N}$ with corresponding inverse marginal utility given by $\left\{I_{n}\right\}_{n=1}^{N}$. Suppose that each $u_{n}$ exhibits $K_{n}$-fund separation. Define another utility function $u$, whose inverse marginal utility is given by

$$
I(\xi)=\sum_{n=1}^{N} t_{n} I_{n}(\xi)
$$

for some non-zero constants $t_{1}, t_{2}, \cdots, t_{N}$. Then, $u$ satisfies $K$-fund separation with

$$
K \leq \sum_{n=1}^{N} K_{n}
$$

where the equality holds if the separating funds of all $u_{n}$ are linearly independent.

Proof of Theorem 2: For each $n$, since $u_{n}$ exhibits $K_{n}$-fund separation, there must exist $\left\{f_{n, k}(\cdot)\right\}_{k}$ and $\left\{\alpha_{n, k}(\cdot)\right\}_{k}$ such that

$$
I_{n}(\lambda \rho)=\sum_{k=1}^{K_{n}} \alpha_{n, k}(\lambda) f_{n, k}(\rho)
$$


By (15), we thus have

$$
I(\lambda \rho)=\sum_{n=1}^{N} t_{n} I_{n}(\lambda \rho)=\sum_{n=1}^{N} \sum_{k=1}^{K_{n}} t_{n} \alpha_{n, k}(\lambda) f_{n, k}(\rho) .
$$

This implies that $u$ satisfies fund separation.

If all $\left\{f_{n, k}(\rho)\right\}_{n, k}$ are linearly independent, then Theorem 1 implies that all $\left\{\alpha_{n, k}(\lambda)\right\}_{n, k}$ are also linearly independent. Hence, $\left\{f_{n, k}(\rho)\right\}_{n, k}$ serve as a set of separating funds for $u$ with the associated investment weights given by $\left\{t_{n} \alpha_{n, k}(\lambda)\right\}_{n, k}$, and the degree of separation is $K=\sum_{n=1}^{N} K_{n}$. If $\left\{f_{n, k}(\rho)\right\}_{n, k}$ are linearly dependent, then multiple funds can be combined, which reduces the degree of separation, i.e., $K<\sum_{n=1}^{N} K_{n}$

Theorem 2 implies that one can generate high-degree separating preferences by taking linear combinations of lower-degree ones in the inverse marginal utility function. One would wonder if this allows us to find the entire set of separating utility based on one-fund separation only. Unfortunately, this is not true, due to the existence of repeated and complex roots of (14), which correspond to logarithm and trigonometric terms in the characterization (3). To illustrate this, it is useful to review one- and two-fund separation here.

\subsubsection{One-Fund Separation}

It is immediate from Theorem (1) that a utility function $u$ exhibits one-fund separation if and only (14) has a single real root $\gamma$, in which case the inverse marginal utility can be written as

$$
I(\xi)=C \xi^{-1 / R},
$$

for some constant $C$ and $R=-1 / \gamma$. Note that changing the sign of $C$ changes preferences, while changing the magnitude without changing the sign only changes the scaling of the utility function and does not change preferences. Conditions for strict concavity are at least implicit in the literature (except perhaps for the mirror CRRA case discussed below), but we repeat them for completeness. Strict concavity requires $I^{\prime}(\xi)=-\frac{C}{R} \xi^{-1-1 / R}<0$, i.e.,

$$
\frac{C}{R}>0
$$


One can verify that the utility function for the one-fund separating preferences is

$$
u(x)=\left\{\begin{array}{ll}
\frac{C}{1-R}\left(\frac{x}{C}\right)^{1-R}, & R \neq 0 \text { or } 1 \\
C \log \left(\frac{x}{C}\right), & R=1
\end{array},\right.
$$

where the domain of consumption is $x \in(0,+\infty)$ when $R>0$ and the domain is $x \in(-\infty, 0)$ when $R<0$. Notice that when $R>0$ and $C>0$, this corresponds to the CRRA utility function, which is defined on positive consumption levels, as in Example 1. When $R<0$ and $C<0$, we refer to this utility function as mirror CRRA preferences, since it has the same form as CRRA but is defined on negative wealth.

\subsubsection{Two-Fund Separation}

If a utility function $u$ exhibits two-fund separation, then Theorem (1) implies that there are potentially four different types of cases, only three of which are consistent with concavity, and only one of which can be constructed by combining one-fund separating preferences.

Case 1: When (14) has two non-zero distinct real roots, $\gamma_{1}$ and $\gamma_{2},(3)$ is equivalent to

$$
I(\xi)=C_{1} \xi^{-1 / R_{1}}+C_{2} \xi^{-1 / R_{2}}
$$

where $R_{1}=-1 / \gamma_{1} \neq R_{2}=-1 / \gamma_{2}$, and $C_{1}$ and $C_{2}$ are arbitrary constants such that $C_{1} / R_{1}>0$ and $C_{2} / R_{2}>0$ for concavity. An example of this case is the SAHARA utility function obtained when $R_{1}=a$ and $R_{2}=-a$ (Example 3 ). For all preferences characterized by (18), the two-fund separating utility function can be obtained as in Theorem 2 from the one-fund separating utility functions with relative risk aversion $R_{1}$ and $R_{2}$.

Case 2: When (14) has two distinct real roots, 0 and $\gamma,(3)$ is equivalent to

$$
I(\xi)=C_{1}+C_{2} \xi^{-1 / R}
$$

where $R=-1 / \gamma$, and $C_{1}$ and $C_{2}$ are arbitrary constants such that $C_{1} \neq 0$ and $C_{2} / R>0$. This can be viewed as the limit of Case 1 when one of the risk aversion levels goes to infinity. An example of this is quadratic utility (Example 2), obtained by adding a constant to a mirror CRRA preference in the inverse marginal utility. All utility functions of this form are in the HARA class and have money separation 
(see Section 3). These preferences cannot be derived by combining preferences with one-fund separation, except as a limiting case.

Case 3: When (14) has two repeated real roots, (3) is equivalent to

$$
I(\xi)=C_{1} \xi^{\gamma}+C_{2} \xi^{\gamma} \log \xi
$$

for some constants $C_{1}$ and $C_{2}$. Strict concavity implies $\gamma=0$ (see Proposition 2 of Section 5), so we must have

$$
I(\xi)=C_{1}+C_{2} \log \xi
$$

which is CARA utility $u(x)=-e^{-A x}$ with $A=-1 / C_{2}>0$ (see Example 4). This case cannot be derived by combining preferences with one-fund separation, because the logarithm term never shows up in one-fund separation.

Case 4: When (14) has a pair of complex roots, (3) is equivalent to

$$
I(\xi)=C_{1} \xi^{\gamma} \cos (b \log \xi)+C_{2} \xi^{\gamma} \sin (b \log \xi)
$$

with constants $b \neq 0, C_{1}$ and $C_{2}$. We will show in Proposition 2 of Section 5 that this form cannot exist under strict concavity.

While the trigonometric terms cannot appear in one- or two-fund separation, they can appear in higher-degree separation, as in Example 5.

\section{Money Separation}

Money separation is a special case of mutual fund separation, which obtains when we can choose the riskless asset as one of the separating funds. Examples of money separation we have encountered so far include quadratic (Example 2) and CARA (Example 4) preferences. In this section, we discuss money separation and its properties in greater depth.

\subsection{Money Separation and Constant Investment Weight}

An interesting observation is that for the quadratic and CARA preferences, both of which exhibit money separation, the optimal investment strategy always involves assigning a constant weight (dollar amount) to one of the separating funds, regardless of the initial wealth level. In particular, for the quadratic case, the constant weight is 
assigned to the riskless asset, whereas a CARA investor optimally assigns a constant weight to a risky fund. A natural question is whether this is merely a coincidence or it actually reveals a property of money separation. The following theorem answers this question.

Theorem 3 A separating utility function exhibits money separation if and only if the optimal investment portfolio can be constructed by assigning a constant amount to one separating fund, regardless of the initial wealth.

Proof of Theorem 3: It is our task to show that having the riskless asset in the span of the separating funds is equivalent to being able to choose a separating fund with a constant investment. We will show that both are equivalent to having a root of equation (14) with $\left(\gamma_{k}, b_{k}\right)=(0,0)$.

By Theorem 1, if a utility function $u$ exhibits mutual fund separation, then the separating funds can be chosen as

$$
f_{k, l}(\rho)=\rho^{\gamma_{k}}(\log \rho)^{l}
$$

when $b_{k}=0$, or

$$
\begin{aligned}
& f_{k, 1, l}(\rho)=\rho^{\gamma_{k}} \cos \left(b_{k} \log \rho\right)(\log \rho)^{l}, \\
& f_{k, 2, l}(\rho)=\rho^{\gamma_{k}} \sin \left(b_{k} \log \rho\right)(\log \rho)^{l} .
\end{aligned}
$$

when $b_{k} \neq 0$ for all $(k, l)$. Therefore, $u$ satisfies money separation if and only if $(20)$, (21), (22), or any of their linear combinations equals a constant. Since any finite set of terms for different $(k, l)$ is linearly independent, money separation holds if and only if $(20)$ is a constant for some $(k, l)$, which happens if and only if $\left(\gamma_{k}, b_{k}\right)=(0,0)$ with $l=0$.

For a separating preference, the investment weights associated with separating funds (20), (21) and (22) are given by

$$
\alpha_{k, l}(\lambda)=\lambda^{\gamma_{k}} \sum_{j=l}^{d_{k, 1}} C_{k, 1, j}\left(\begin{array}{l}
j \\
l
\end{array}\right)(\log \lambda)^{j-l}
$$


when $b_{k}=0$, and

$$
\begin{aligned}
\alpha_{k, 1, l}(\lambda)= & \mathbf{1}_{l \leq d_{k, 1}} \lambda^{\gamma_{k}} \cos \left(b_{k} \log \lambda\right) \sum_{j=l}^{d_{k, 1}} C_{k, 1, j}\left(\begin{array}{c}
j \\
l
\end{array}\right)(\log \lambda)^{j-l} \\
& +\mathbf{1}_{l \leq d_{k, 2}} \lambda^{\gamma_{k}} \sin \left(b_{k} \log \lambda\right) \sum_{j=l}^{d_{k, 2}} C_{k, 2, j}\left(\begin{array}{l}
j \\
l
\end{array}\right)(\log \lambda)^{j-l}, \\
\alpha_{k, 2, l}(\lambda)= & \mathbf{1}_{l \leq d_{k, 2}} \lambda^{\gamma_{k}} \cos \left(b_{k} \log \lambda\right) \sum_{j=l}^{d_{k, 2}} C_{k, 2, j}\left(\begin{array}{c}
j \\
l
\end{array}\right)(\log \lambda)^{j-l} \\
& -\mathbf{1}_{l \leq d_{k, 1}} \lambda^{\gamma_{k}} \sin \left(b_{k} \log \lambda\right) \sum_{j=l}^{d_{k, 1}} C_{k, 1, j}\left(\begin{array}{l}
j \\
l
\end{array}\right)(\log \lambda)^{j-l}
\end{aligned}
$$

when $b_{k} \neq 0$. Therefore, one separating fund receives a constant weight if and only if (23), (24), (25) or any of their linear combinations equals a constant. Since any finite set of terms for different $(k, l)$ is linearly independent, a constant weight obtains if and only if $(23)$ is a constant for some $(k, l)$, which happens if and only if $\left(\gamma_{k}, b_{k}\right)=(0,0)$ with $l=d_{k, 1}$.

Hence, the theorem is proven.

According to Theorem 3, for a money separating preference, the optimal investment strategy can be constructed by assigning a constant amount to one of the separating funds. Interestingly, the fund with the constant weight can be either the riskless asset or a risky fund. As shown in the above proof, the riskless asset corresponds to $\left(\gamma_{k}, b_{k}\right)=(0,0)$ and $l=0$, whereas the constant investment weight is assigned to the separating fund with $\left(\gamma_{k}, b_{k}\right)=(0,0)$ and $l=d_{k, 1}$. Therefore, the riskless asset receives a constant weight if and only if $\left(\gamma_{k}, b_{k}\right)=(0,0)$ and $d_{k, 1}=0$, i.e., (14) has a non-repeated zero root, as in the case of quadratic utility. When the zero root is repeated, as for CARA utility, then the riskless asset receives a wealthdependent investment weight, and the constant weight is assigned to a risky fund.

\subsection{Money Separation and Shifts in Utility}

In this section, we show that money separation is closely related to shifts in the utility function. Indeed, we can construct money separating preferences from non-money separation by introducing a shift in the utility function. This is formalized in the following theorem. 
Theorem 4 Suppose that a utility function u exhibits $K$-fund non-money separation. Define $\hat{u}(x)=u\left(x-C_{0}\right)$ for an arbitrary constant $C_{0} \neq 0$. Then, $\hat{u}$ satisfies $(K+1)$ fund money separation.

Proof of Theorem 4: Since

$$
\hat{u}(x)=u\left(x-C_{0}\right),
$$

one can verify that the associated inverse marginal utility satisfies

$$
\hat{I}(\xi)=I(\xi)+C_{0}
$$

Thus, introducing a shift to the utility function is equivalent to adding a constant term in the inverse marginal utility.

If $u$ exhibits $K$-fund non-money separation, then there exist $\alpha_{k}(\lambda)$ and $f_{k}(\rho)$ for $k=1,2, \cdots, K$ such that

$$
I(\lambda \rho)=\sum_{k=1}^{K} \alpha_{k}(\lambda) f_{k}(\rho)
$$

where none of $f_{k}(\rho)$ or any of their linear combination equals a constant, and none of $\alpha_{k}(\lambda)$ or any of their linear combination equals a constant.

From (26) and (27), we have

$$
\hat{I}(\lambda \rho)=I(\lambda \rho)+C_{0}=\sum_{k=1}^{K} \alpha_{k}(\lambda) f_{k}(\rho)+C_{0} .
$$

Hence, $\hat{u}$ exhibits $(K+1)$-fund money separation with the $K+1$ separating funds given by $\left\{f_{k}(\rho)\right\}_{k}$ and 1 , and the associated investment weights given by $\left\{\alpha_{k}(\lambda)\right\}_{k}$ and $C_{0}$.

To demonstrate how Theorem 4 works, it is useful to consider the one-fund separating preferences

$$
u(x)=\left\{\begin{array}{ll}
\frac{C}{1-R}\left(\frac{x}{C}\right)^{1-R}, & R \neq 0 \text { or } 1 \\
C \log \left(\frac{x}{C}\right), & R=1
\end{array},\right.
$$

whose inverse marginal utility function is given by

$$
I(\xi)=C \xi^{-1 / R}
$$


By introducing a shift to the utility function, we obtain the HARA class

$$
\hat{u}(x)=\left\{\begin{array}{ll}
\frac{C}{1-R}\left(\frac{x-C_{0}}{C}\right)^{1-R}, & R \neq 0 \text { or } 1 \\
C \log \left(\frac{x-C_{0}}{C}\right), & R=1
\end{array},\right.
$$

with inverse marginal utility function

$$
\hat{I}(\xi)=C \xi^{-1 / R}+C_{0}
$$

We have seen in Section 2.4.2 that these preferences satisfy two-fund money separation when $C_{0} \neq 0$. Hence, we obtain two-fund money separating preferences by introducing a non-zero shift to the one-fund separating utility function.

It is worth mentioning that money separation obtained this way always assigns a constant weight to the riskless asset. As discussed in Section 3.1, this is only one of the two possible cases of money separation. The other case in which the constant weight is assigned to a risky fund cannot be obtained using this approach.

\section{Closed-Form Utility Functions}

We have characterized the set of separating preferences in terms of the inverse marginal utility function. We then ask whether we are able to derive the associated utility function. A natural way to do this is by first inverting $I$ to obtain $u^{\prime}$, and then integrating $u^{\prime}$ to obtain $u$. Unfortunately, however, this does not yield a closed-form expression except in a few special cases, with those already in the literature limited to one- and two-fund separation (e.g., CRRA, CARA and quadratic utility, etc.). In this section, we discuss two classes of three-fund money separating preferences, SAHARA and GOBI utility, for which closed-form expressions of the utility function exist.

\subsection{SAHARA Utility}

The SAHARA preferences are proposed by Chen, Pelsser and Vellekoop (2011). The standard SAHARA utility is defined on the entire real line $\mathbb{R}$ and has an inverse marginal utility function of the form

$$
I(\xi)=\frac{1}{2}\left(\xi^{-\frac{1}{a}}-b^{2} \xi^{\frac{1}{a}}\right),
$$

with $a, b>0$. As shown in Example 3, these preferences exhibit two-fund non-money separation. 
More generally, we allow for a shift in the SAHARA utility function, which is equivalent to adding a constant term to the inverse marginal utility, i.e.,

$$
I(\xi)=\frac{1}{2}\left(\xi^{-\frac{1}{a}}-b^{2} \xi^{\frac{1}{a}}\right)+C_{0} .
$$

The constant $C_{0}$ is referred to as the default point in Chen, Pelsser and Vellekoop (2011), and the standard form obtains by setting $C_{0}=0$. We know from Theorem 4 that when $C_{0} \neq 0$, the shifted SAHARA utility exhibits three-fund money separation. The separating funds can be chosen as

$$
\begin{aligned}
& f_{1}(\rho)=\rho^{-\frac{1}{a}}, \\
& f_{2}(\rho)=\rho^{\frac{1}{a}}, \\
& f_{3}(\rho)=1,
\end{aligned}
$$

with the corresponding investment weights given by

$$
\begin{aligned}
\alpha_{1}(\lambda) & =\frac{1}{2} \lambda^{-\frac{1}{a}}, \\
\alpha_{2}(\lambda) & =-\frac{1}{2} b^{2} \lambda^{\frac{1}{a}}, \\
\alpha_{3}(\lambda) & =C_{0} .
\end{aligned}
$$

One nice property of the SAHARA utility is that the two power terms in the inverse marginal utility, $\xi^{-\frac{1}{a}}$ and $\xi^{\frac{1}{a}}$, are reciprocals of each other. We can thus rewrite (29) as a quadratic equation of $\xi^{\frac{1}{a}}$, which further allows us to invert $I$ to recover the underlying utility function. Specifically, multiplying $\xi^{\frac{1}{a}}$ on both sides of (29) and setting $I(\xi)=x$ and $\xi=u^{\prime}(x)$ yield

$$
b^{2}\left(u^{\prime}(x)\right)^{\frac{2}{a}}+2\left(x-C_{0}\right)\left(u^{\prime}(x)\right)^{\frac{1}{a}}-1=0,
$$

which can be viewed as a quadratic equation of $\left(u^{\prime}(x)\right)^{\frac{1}{a}}$. Since $u^{\prime}(x)>0$, we focus on positive solutions to (30). Using the properties of quadratic equations, we can verify that (30) has a unique positive solution given by

$$
u^{\prime}(x)=\left(\frac{\sqrt{(G(x))^{2}+b^{2}}-G(x)}{b^{2}}\right)^{a},
$$

where $G(x)=x-C_{0}$. Integrating $u^{\prime}(x)$ then gives us the utility function

$$
u(x)=\left\{\begin{array}{ll}
\frac{1}{1-a^{2}}\left(\sqrt{(G(x))^{2}+b^{2}}+G(x)\right)^{-a}\left(a \sqrt{(G(x))^{2}+b^{2}}+G(x)\right), & a \neq 1 \\
\frac{1}{2} \log \left(\sqrt{(G(x))^{2}+b^{2}}+G(x)\right)+\frac{G(x)}{2 b^{2}}\left(\sqrt{(G(x))^{2}+b^{2}}-G(x)\right), & a=1
\end{array} .\right.
$$




\subsection{GOBI Utility}

Define the class of GOBI preferences, whose inverse marginal utility function takes the form

$$
I(\xi)=C_{1} \xi^{\gamma}+C_{2} \xi^{2 \gamma}+C_{0},
$$

where $\gamma, C_{1}$ and $C_{2}$ are non-zero constants such that $C_{1} \gamma<0$ and $C_{2} \gamma<0$ for concavity. As for the SAHARA class, we include a constant $C_{0}$ in the inverse marginal utility to capture potential shifts in the utility function. As opposed to the SAHARA utility, which is defined on the entire real line, the GOBI utility is defined on a half real line. In particular, the domain is $x \in\left(C_{0},+\infty\right)$ when $\gamma<0$, and the domain is $x \in\left(-\infty, C_{0}\right)$ when $\gamma>0$.

It is easy to verify that when $C_{0} \neq 0$, the GOBI preferences have three-fund money separation. The separating funds can be chosen as

$$
\begin{aligned}
& f_{1}(\rho)=\rho^{\gamma}, \\
& f_{2}(\rho)=\rho^{2 \gamma}, \\
& f_{3}(\rho)=1,
\end{aligned}
$$

with the corresponding investment weights given by

$$
\begin{aligned}
& \alpha_{1}(\lambda)=C_{1} \lambda^{\gamma}, \\
& \alpha_{2}(\lambda)=C_{2} \lambda^{2 \gamma}, \\
& \alpha_{3}(\lambda)=C_{0} .
\end{aligned}
$$

When $C_{0}=0$, this reduces to two-fund non-money separation.

As for the SAHARA class, (31) can be viewed as a quadratic equation of $\xi^{\gamma}$. Setting $I(\xi)=x$ and $\xi=u^{\prime}(x)$, we can rewrite $(31)$ as

$$
C_{2}\left(u^{\prime}(x)\right)^{2 \gamma}+C_{1}\left(u^{\prime}(x)\right)^{\gamma}-\left(x-C_{0}\right)=0 .
$$

Since $u^{\prime}(x)>0$, we focus on positive solutions to (32). Using the properties of quadratic equations, we can verify that (32) has a unique positive solution given by

$$
u^{\prime}(x)=\left(\frac{G(x)-C_{1}}{2 C_{2}}\right)^{\frac{1}{\gamma}},
$$


where $G(x)=\sqrt{C_{1}^{2}+4 C_{2}\left(x-C_{0}\right)}$. Integrating $u^{\prime}(x)$ then gives us the utility function

$$
u(x)=\left\{\begin{array}{ll}
\frac{\gamma}{(2 \gamma+1)\left(2 C_{2}\right)^{1+1 / \gamma}}\left(G(x)-C_{1}\right)^{1+1 / \gamma}\left(G(x)+\frac{\gamma}{\gamma+1} C_{1}\right), & \gamma \neq-\frac{1}{2},-1 \text { or } 0 \\
\frac{1}{3}\left(\frac{2}{C_{2}}\right)^{1 / 2}\left(G(x)-C_{1}\right)^{1 / 2}\left(G(x)+2 C_{1}\right), & \gamma=-\frac{1}{2} \\
G(x)+C_{1} \log \left(G(x)-C_{1}\right), & \gamma=-1
\end{array} .\right.
$$

\section{Strict Concavity}

We have so far assumed that all utility functions under consideration are strictly concave, i.e., $u^{\prime \prime}<0$. This condition allows us to characterize the set of separating preferences by (3) in terms of the inverse marginal utility. However, not all functions satisfying (3) are strictly concave for all parameter values. In this section, we study strict concavity of separating preferences and how it can be used to narrow down our separating class.

Strict concavity is equivalent to $I^{\prime}(\xi) \leq 0$ on the relevant range. Given the functional form of our separating class $(3)$, the zeros of $I^{\prime}(\xi)$ can only be isolated. While the necessary and sufficient condition for strict concavity turns out to be straightforward for one- and two-fund separation, it can become very complicated, if not impossible, when higher-degree separation is taken into account. To see this, consider as an example a $K$-fund separating preference whose inverse marginal utility takes the form

$$
I(\xi)=\sum_{k=1}^{K} C_{k} \xi^{\gamma_{k}},
$$

with non-zero $C_{k}$. A sufficient condition for concavity is that $C_{k} \gamma_{k}<0$ for all $k$, but this condition is not necessary. In $(33), I(\xi)$ is like a polynomial (and is a polynomial if all $\gamma_{k}$ are integer multiples of the same number). For a polynomial, the utility function is concave if $I^{\prime}(\xi)$ is negative somewhere and all positive roots of $I^{\prime}(\xi)$ have even order. Even finding the roots is a hard problem for which there is no simple characterization. Other cases combining power terms with trigonometric functions and/or logarithms are even harder. Therefore, it seems impossible to come close to a complete characterization of concavity.

Despite the intrinsic challenge in providing a necessary and sufficient characterization, below we seek to identify some necessary conditions on the parameter values that are needed to induce strict concavity. While these conditions are not sufficient, 
they allow us to narrow down the class of separating preferences by ruling out parameter values and forms of the inverse marginal utility that are not permitted.

The next proposition deals with the special case of fund separation, in which the inverse marginal utility is given by the sum of power terms only.

Proposition 1 Consider a separating preference, whose inverse marginal utility is given by

$$
I(\xi)=\sum_{k=1}^{K} C_{k} \xi^{\gamma_{k}}+C_{0},
$$

with non-zero $C_{1}, C_{2}, \cdots, C_{K}$ and non-zero $\gamma_{1}, \gamma_{2}, \cdots, \gamma_{K}$ such that $\gamma_{1}<\gamma_{2}<\cdots<$ $\gamma_{K}$. Then, strict concavity implies $C_{1} \gamma_{1}<0$ and $C_{K} \gamma_{K}<0$.

The following proposition further rules out forms of the inverse marginal utility that violate strict concavity.

Proposition 2 If a separating utility function is strictly concave, then its inverse marginal utility I cannot take the following forms.

1. $I(\xi)=P(\log \xi)$, where the polynomial function $P(\cdot)$ is of an even degree;

2. $I(\xi)=\xi^{\gamma} P(\log \xi)$, where $\gamma \neq 0$ and the polynomial function $P(\cdot)$ is of an odd degree;

3. $I(\xi)=\sum_{k=1}^{J} \xi^{\gamma_{k}} P_{k, 1}(\log \xi) \cos \left(b_{k} \log \xi\right)+\sum_{k=1}^{J} \xi^{\gamma_{k}} P_{k, 2}(\log \xi) \sin \left(b_{k} \log \xi\right)$, where $b_{k} \neq 0$ for all $k$.

While preferences with an inverse marginal utility function of the forms listed in Proposition 2 violate strict concavity, more complex $I(\xi)$ including these terms can be concave. In fact, we have seen in Example 5 that the trigonometric terms can be consistent with strict concavity when additional terms are present. We further illustrate how to construct functional forms and choose parameter values to meet strict concavity using the following examples.

Example 6 Consider a three-fund separating preference, whose inverse marginal utility function is given by

$$
I(\xi)=\xi^{\gamma}\left(C_{1}+C_{2} \log \xi+C_{3}(\log \xi)^{2}\right),
$$


with non-zero $\gamma$ and $C_{3}$. Differentiating $I$ yields

$$
I^{\prime}(\xi)=\xi^{\gamma-1}\left(\left(\gamma C_{1}+C_{2}\right)+\left(\gamma C_{2}+2 C_{3}\right) \log \xi+\gamma C_{3}(\log \xi)^{2}\right) .
$$

Since $\xi^{\gamma-1}>0$, in order to have $I^{\prime}(\xi) \leq 0$ we only need $\forall \xi>0$,

$$
\left(\gamma C_{1}+C_{2}\right)+\left(\gamma C_{2}+2 C_{3}\right) \log \xi+\gamma C_{3}(\log \xi)^{2} \leq 0 .
$$

Notice that the left-hand side can be viewed as a quadratic function of $\log \xi$. As a result, this inequality holds if and only if

$$
\gamma C_{3}<0
$$

and

$$
\left(\gamma C_{2}+2 C_{3}\right)^{2}-4 \gamma C_{3}\left(\gamma C_{1}+C_{2}\right) \leq 0 .
$$

Therefore, any set of parameter values satisfying the above two conditions (e.g., $\gamma=$ $\left.-1, C_{1}=3, C_{2}=2, C_{3}=1\right)$ would give rise to a strictly concave separating utility function.

Example 7 Consider a three-fund separating preference, whose inverse marginal utility function is given by

$$
I(\xi)=\xi^{\gamma_{1}}\left(C_{1}+C_{2} \log \xi\right)+C_{3} \xi^{\gamma_{2}},
$$

with non-zero $\gamma_{1}, \gamma_{2}, C_{2}, C_{3}$ and $\gamma_{1} \neq \gamma_{2}$. Differentiating I yields

$$
\begin{aligned}
I^{\prime}(\xi) & =\xi^{\gamma_{1}-1}\left(\left(\gamma_{1} C_{1}+C_{2}\right)+\gamma_{1} C_{2} \log \xi\right)+C_{3} \gamma_{2} \xi^{\gamma_{2}-1} \\
& =\xi^{\gamma_{2}-1}\left[\left(\gamma_{1} C_{1}+C_{2}\right) \xi^{\gamma_{1}-\gamma_{2}}+\gamma_{1} C_{2} \xi^{\gamma_{1}-\gamma_{2}} \log \xi+C_{3} \gamma_{2}\right] .
\end{aligned}
$$

Since $\xi^{\gamma_{2}-1}>0$, the sign of $I^{\prime}(\xi)$ depends on that of $\left(\gamma_{1} C_{1}+C_{2}\right) \xi^{\gamma_{1}-\gamma_{2}}+$ $\gamma_{1} C_{2} \xi^{\gamma_{1}-\gamma_{2}} \log \xi+C_{3} \gamma_{2}$. We will show that (i) when $\gamma_{1}<\gamma_{2}, \gamma_{1} C_{1}+C_{2}<0$, and $\gamma_{1} C_{2}>0$ (e.g., $\gamma_{1}=-1, \gamma_{2}=1, C_{1}=1, C_{2}=-1$ ), or (ii) when $\gamma_{1}>\gamma_{2}$, $\gamma_{1} C_{1}+C_{2}<0$, and $\gamma_{1} C_{2}<0$ (e.g., $\gamma_{1}=1, \gamma_{2}=-1, C_{1}=-1, C_{2}=-1$ ), we can always set $C_{3} \gamma_{2}$ low enough such that $I^{\prime}(\xi)<0$ holds for all $\xi>0$. We will show part (i) in detail only. The analysis for part (ii) is parallel.

Under (i), since $\gamma_{1} C_{1}+C_{2}<0$, we have $\left(\gamma_{1} C_{1}+C_{2}\right) \xi^{\gamma_{1}-\gamma_{2}}<0$ for all $\xi>0$. Since $\gamma_{1} C_{2}>0$, we have $\gamma_{1} C_{2} \xi^{\gamma_{1}-\gamma_{2}} \log \xi<0$ for all $\xi<1$ and $\gamma_{1} C_{2} \xi^{\gamma_{1}-\gamma_{2}} \log \xi \geq 0$ for all $\xi \geq 1$. Given $\gamma_{1}<\gamma_{2}$, we know

$$
\lim _{\xi \rightarrow+\infty} \gamma_{1} C_{2} \xi^{\gamma_{1}-\gamma_{2}} \log \xi=0 .
$$


Since $\gamma_{1} C_{2} \xi^{\gamma_{1}-\gamma_{2}} \log \xi$ is continuous, (35) implies that $\gamma_{1} C_{2} \xi^{\gamma_{1}-\gamma_{2}} \log \xi$ must be bounded above. Then, setting $C_{3} \gamma_{2}$ equal to or lower than the negative value of this upper bound is enough to guarantee $I^{\prime}(\xi)<0$ for all $\xi>0$, giving rise to a strictly concave utility function.

\section{Machina Preferences}

Our analyses so far have focused on von Neumann-Morgenstern preferences, i.e., investors have expected utility. Machina (1982) shows that the von Neumann-Morgenstern preferences can actually be viewed as a special case of a larger class of preferences, which we call "Machina preferences". Assume that investors' utility is a function of consumption distribution only and is smooth in the sense of Fréchet differentiability. ${ }^{7}$ Machina proves that such utility functions are locally consistent with expected utility. In this section, we ask whether our fund separation results derived for expected utility can be extended to the Machina preferences.

Consider a Fréchet differentiable utility function $V(\cdot)$ defined over the distribution of consumption. Let $x$ and $x^{*}$ denote two random consumption portfolios with the corresponding cumulative distribution functions given by $F$ and $F^{*}$. Suppose that $F$ and $F^{*}$ lie very close to each other. Machina (1982) shows

$$
V(F)-V\left(F^{*}\right) \approx \int U\left(z ; F^{*}\right)\left(d F-d F^{*}\right)=\mathbf{E}\left[U\left(x ; F^{*}\right)\right]-\mathbf{E}\left[U\left(x^{*} ; F^{*}\right)\right],
$$

or equivalently,

$$
V(F) \approx V\left(F^{*}\right)+\mathbf{E}\left[U\left(x ; F^{*}\right)\right]-\mathbf{E}\left[U\left(x^{*} ; F^{*}\right)\right]
$$

where $U(z ; F)$ is the local utility function over consumption level $z$ evaluated at distribution $F$. Assume that $U(\cdot ; F)$ is strictly concave for all $F$. It can be learned from (36) that the Machina preferences can be modeled locally as expected utility.

With Machina preferences, investors face the following utility maximization problem.

\footnotetext{
${ }^{7}$ Fréchet differentiability is an infinite-dimensional version of differentiability. The idea is that the utility function changes smoothly with the distribution of consumption. We are being informal about the topology used to define the Fréchet derivative if consumption is not bounded. In Machina's original work (as in many derivations of von Neumann-Morgenstern preferences), it is assumed that consumption is bounded. To formalized what we are doing for unbounded consumptions, we would have to specify the topology over distribution functions to define the sense of approximation.
} 
Problem 2 Choose consumption $x$ to

$$
\max _{x} V(F)
$$

subject to the budget constraint

$$
\mathbf{E}(x \rho) \leq w_{0} .
$$

Suppose that $x^{*}$ solves Problem 2. Then, it must maximize (36) with local utility function $U\left(\cdot ; F^{*}\right)$ evaluated at $F^{*}$. Fixing $F^{*}$, both $V\left(F^{*}\right)$ and $\mathbf{E}\left[U\left(x^{*} ; F^{*}\right)\right]$ are constants. Therefore, $x^{*}$ maximizes $\mathbf{E}\left[U\left(x ; F^{*}\right)\right]$. Given strict concavity of $U(\cdot ; F)$, $x^{*}$ must be unique, and it also solves the following problem.

Problem 3 Choose consumption $x$ to

$$
\max _{x} \mathbf{E}\left[U\left(x ; F^{*}\right)\right]
$$

subject to the budget constraint

$$
\mathbf{E}(x \rho) \leq w_{0} .
$$

It seems that we are faced with a similar problem as in the case of von NeumannMorgenstern preferences. Apparently, if all local utility functions $U(\cdot ; F)$ at all $F$ satisfy fund separation with the same separation funds, then $V(\cdot)$ exhibits fund separation globally. In fact, this condition is stronger than needed. The only thing we need is for all optimal consumption portfolios corresponding to all possible initial wealth levels to be spanned by the same set of separating funds. Since each optimal consumption portfolio corresponds to a different local utility function, we only need fund separation for each local utility function $U(\cdot ; F)$ at the particular wealth level supporting $F$ as the optimal consumption portfolio. Formally, let $\mathcal{U}_{F} \equiv\{U(\cdot ; F), \forall F\}$ denote the set of local utility functions $U(\cdot ; F)$ evaluated at all possible $F$. Then, we have the following sufficient but not necessary condition for fund separation for Machina preferences.

Theorem 5 Consider a Machina utility function $V(\cdot)$, with the associated set of local utility functions at all consumption distributions given by $\mathcal{U}_{F}$. If $\mathcal{U}_{F}$ satisfies mutual fund separation (characterized by Corollary 1), then $V(\cdot)$ satisfies mutual fund separation. 
To illustrate fund separation for Machina preferences, we now provide two examples, one of which meets the sufficient condition of Theorem 5 and the other of which does not. However, we will see that both examples satisfy two-fund separation. The first example is the mean-variance preferences, which have originally been shown to satisfy two-fund money separation in Tobin (1958).

Example 8 Consider the mean-variance preferences

$$
V(F)=\mathbf{E}(x)-\frac{a}{2} \mathbf{E}\left[(x-\mathbf{E}(x))^{2}\right]=\mathbf{E}(x)-\frac{a}{2}\left[\mathbf{E}\left(x^{2}\right)-(\mathbf{E}(x))^{2}\right],
$$

with $a>0$. This can equivalently be rewritten as

$$
V(F)=\int_{x=-\infty}^{+\infty}\left(x-\frac{a}{2} x^{2}\right) d F+\frac{a}{2}\left(\int_{x=-\infty}^{+\infty} x d F\right)^{2} .
$$

Differentiating $V$ with respect to $F$ gives the local utility function

$$
U(x ; F)=\left(x-\frac{a}{2} x^{2}\right)+a x\left(\int_{x=-\infty}^{+\infty} x d F\right)=-\frac{a}{2} x^{2}+[1+a \mathbf{E}(x)] x .
$$

Fixing any $F, \mathbf{E}(x)$ is a constant, and thus (37) is a quadratic function. This implies that the local utility functions at all $F$ are quadratic. We already know from Example 2 that all quadratic utility funtions satisfy two-fund money separation with the same separating funds. It is then immediate from Theorem 5 that the mean-variance preferences exhibit two-fund money separation.

The mean-variance preferences assume that the only risk that investors are averse to is the variance of consumptions. As a second example, we incorporate an additional dimension of risk into the utility function, which is the downside risk defined as $\mathbf{E}\left[\left([x-\mathbf{E}(x)]^{-}\right)^{2}\right]$, where $[\cdot]^{-}=\min (0, \cdot)$. Downside risk is originally introduced in Bawa and Lindenberg (1977). Kadan, Liu and Liu (2016) show that when the risk measure is the sum of variance and downside risk, then the resulting mean-risk preferences satisfy two-fund money separation.

Example 9 Consider the mean-risk preferences

$$
V(F)=\mathbf{E}(x)-\frac{a}{2} \mathbf{E}\left[(x-\mathbf{E}(x))^{2}\right]-\frac{b}{2} \mathbf{E}\left[\left([x-\mathbf{E}(x)]^{-}\right)^{2}\right],
$$

where risk has a variance component and a downside risk component with $a, b>0$. This can equivalently be rewritten as

$$
V(F)=\int_{x=-\infty}^{+\infty}\left(x-\frac{a}{2} x^{2}\right) d F+\frac{a}{2}\left(\int_{x=-\infty}^{+\infty} x d F\right)^{2}-\frac{b}{2} \int_{x=-\infty}^{\mu(F)}[x-\mu(F)]^{2} d F,
$$


where $\mu(F)=\int_{x=-\infty}^{+\infty} x d F$ is the expected consumption given distribution function $F$. Differentiating $V$ with respect to $F$ gives the local utility function

$U(x ; F)=-\frac{a}{2} x^{2}+[1+a \mathbf{E}(x)] x-\frac{b}{2}[x-\mu(F)]^{2} \mathbf{1}_{x<\mu(F)}+b x \int_{x=-\infty}^{\mu(F)}[x-\mu(F)] d F$,

where $\mathbf{1}_{x<\mu(F)}$ is an indicator function that takes a value of 1 if $x<\mu(F)$ and 0 otherwise. Apparently, (37) does not belong to the class of separating utility functions characterized by (3). Hence, in this case the local utility function does not satisfy fund separation.

While the sufficient condition in Theorem (5) fails, fund separation still holds in this case. To see this, taking the first order condition of (38) with respect to $x$ subject to the budget constraint yields

$$
1-a\left[x^{*}-\mathbf{E}\left(x^{*}\right)\right]-b\left[x^{*}-\mathbf{E}\left(x^{*}\right)\right]^{-}=\lambda \rho .
$$

Solving for the optimal consumption gives

$$
x^{*}=\mathbf{E}\left(x^{*}\right)+\max \left(\frac{1-\lambda \rho}{a}, \frac{1-\lambda \rho}{a+b}\right) .
$$

It can be verified that fixing the distribution of $\rho, \lambda$ is a constant, whereas $\mathbf{E}\left(x^{*}\right)$ depends on the initial wealth $w_{0}$. Therefore, $V(F)$ satisfies two-fund money separation with separating funds

$$
\begin{aligned}
& f_{1}(\rho)=1, \\
& f_{2}(\rho)=\max \left(\frac{1-\lambda \rho}{a}, \frac{1-\lambda \rho}{a+b}\right) .
\end{aligned}
$$

The corresponding investment weights are given by

$$
\begin{aligned}
& \alpha_{1}\left(w_{0}\right)=\mathbf{E}\left(x^{*}\right), \\
& \alpha_{2}\left(w_{0}\right)=1 .
\end{aligned}
$$

\section{Conclusion}

This paper extends Cass and Stiglitz (1970) and studies the general preference-based $K$-fund separation. We provide a complete characterization of separating preferences in terms of the inverse marginal utility function and demonstrate our results using various examples. We show how a subset of high-degree separating preferences can 
be constructed by adding low-degree separation in the inverse marginal utility. We use one- and two-fund separation to illustrate cases that can be constucted this way and cases that cannot.

We also study money separation, for which we can choose the riskless asset as one of the separating funds. We show that money separation holds if and only if we can choose a separating fund whose optimal investment amount is constant and does not depend on the initial wealth. Somewhat surprisingly, the constant investment can be assigned to either the riskless asset or a risky fund. We show that money separation is closely related to shifts in the utility function. Starting with a non-money separating preference, one can construct money separation by introducing a non-zero shift in the utility function.

Our characterization provides us with a rich set of preferences satisfying fund separation and helps motivate new functional forms of utility functions that are tractable and have interesting properties. In particular, we provide two classes of three-fund separating preferences, SAHARA and GOBI, for which closed-form expressions of the utility function can be derived. These preference can be very useful for theoretical modeling and empirical tests in future research.

\section{Appendix}

As preparation for the formal proof of Theorem 1, we now review the concept of Wronkian, which is first introduced by Józef Hoene-Wronski (1812) and named by Thomas Muir (1882).

Let $\Phi(\lambda)=\left(\phi_{1}(\lambda), \phi_{2}(\lambda), \cdots, \phi_{K}(\lambda)\right)$ denote a vector of functions with all $\phi_{k}(\lambda)$ defined over a real interval $\Lambda \subset \mathbb{R}$ with at least $K-1$ finite continuous derivatives. Then the Wronskian of $\Phi$ at $\lambda$ is defined as

$$
W[\Phi](\lambda)=\left|\begin{array}{ccc}
\phi_{1}(\lambda) & \cdots & \phi_{K}(\lambda) \\
\phi_{1}^{(1)}(\lambda) & \cdots & \phi_{K}^{(1)}(\lambda) \\
\vdots & \ddots & \vdots \\
\phi_{1}^{(K-1)}(\lambda) & \cdots & \phi_{K}^{(K-1)}(\lambda)
\end{array}\right| .
$$

It is apparent that if $\Phi(\lambda)$ is linearly dependent on $\Lambda$, we have $W[\Phi](\lambda)=0$ for all $\lambda \in \Lambda$. Somewhat surprisingly, however, the reverse does not hold true generally. That is, the identical vanishing of the Wronskian does not necessarily imply linear dependence of $\Phi(\lambda)$. Nevertheless, Bôcher (1900) shows that this is indeed true when 
all $\phi_{k}(\lambda)$ are analytic functions.

Proposition 3 If all $\phi_{k}(\lambda)$ are analytic functions on $\Lambda$, then $W[\Phi](\lambda)=0$ for all $\lambda \in \Lambda$ if and only if $\Phi(\lambda)$ is linearly dependent on $\Lambda$.

Proof of Theorem 1: ( $K$-fund separation implies (3)) The sketch of the proof in the text shows that when $M_{0}(\lambda)$ given by (10) is invertible at some $\lambda$, a utility function exhibiting $K$-fund separation must satisfy (3). To prove that (3) is necessary, we only need to show that (3) is obtained even when $M_{0}(\lambda)$ is not invertible at any $\lambda$.

Suppose $M_{0}(\lambda)$ is not invertible at any $\lambda$. Consider multiplying (2) by $\lambda^{j}$ for $j=0,1, \cdots, K$, i.e.,

$$
\lambda^{j} I(\lambda \rho)=\sum_{k=1}^{K} \lambda^{j} \alpha_{k}(\lambda) f_{k}(\rho),
$$

where $j=0$ corresponds to the baseline case explored in the sketch of the proof. Taking derivatives with respect to $\lambda$ yields

$$
\left(\begin{array}{c}
\frac{\partial\left[\lambda^{j} I(\lambda \rho)\right]}{\partial \lambda} \\
\vdots \\
\frac{\partial^{K}\left[\lambda^{j} I(\lambda \rho)\right]}{\partial \lambda^{K}}
\end{array}\right)=M_{j}(\lambda)\left(\begin{array}{c}
f_{1}(\rho) \\
\vdots \\
f_{K}(\rho)
\end{array}\right)
$$

where

$$
M_{j}(\lambda)=\left(\begin{array}{ccc}
\frac{\partial\left[\lambda^{j} \alpha_{1}(\lambda)\right]}{\partial \lambda} & \cdots & \frac{\partial\left[\lambda^{j} \alpha_{K}(\lambda)\right]}{\partial \lambda} \\
\vdots & \ddots & \vdots \\
\frac{\partial^{K}\left[\lambda^{j} \alpha_{1}(\lambda)\right]}{\partial \lambda^{K}} & \cdots & \frac{\partial^{K}\left[\lambda^{j} \alpha_{K}(\lambda)\right]}{\partial \lambda^{K}}
\end{array}\right)
$$

If $M_{j}(\lambda)$ is invertible at some $\lambda$ for some $j$, we can follow the same procedure as in the sketch of the proof. Specifically, we solve for $f_{k}(\rho)$ as functions of $I(\lambda \rho)$ and its derivatives, and plug them back into (2). Rearranging terms and evaluating at $\lambda=1$ (or at any positive value of $\lambda$ where $\left(M_{j}(\lambda)\right)^{-1}$ exists) give us a differential equation of the form (13). Then, solving (13) for $I(\xi)$ again leads to (3).

Now suppose $M_{j}(\lambda)$ is not invertible at any $\lambda$ for any $j=0,1, \cdots, K$, i.e., $\left|M_{j}(\lambda)\right|=0$ for all $\lambda$ and $j$. Notice that $\left|M_{j}(\lambda)\right|$ is the Wronskian of $\left(\frac{\partial\left[\lambda^{j} \alpha_{1}(\lambda)\right]}{\partial \lambda}, \cdots, \frac{\partial\left[\lambda^{j} \alpha_{K}(\lambda)\right]}{\partial \lambda}\right)$. Since $\alpha_{k}(\lambda)$ are analytic functions, so are $\frac{\partial\left[\lambda^{j} \alpha_{k}(\lambda)\right]}{\partial \lambda}$. By Proposition $3,\left(\frac{\partial\left[\lambda^{j} \alpha_{1}(\lambda)\right]}{\partial \lambda}, \cdots, \frac{\partial\left[\lambda^{j} \alpha_{K}(\lambda)\right]}{\partial \lambda}\right)$ is linearly dependent for each $j$. In other words, for each $j$ there exist $t_{1}^{j}, t_{2}^{j}, \cdots, t_{K}^{j}$ 
not all equal to zero such that

$$
\sum_{k=1}^{K} t_{k}^{j} \frac{\partial\left[\lambda^{j} \alpha_{k}(\lambda)\right]}{\partial \lambda}=0 .
$$

Integrating with respect to $\lambda$ and dividing by $\lambda^{j}$ yield

$$
\sum_{k=1}^{K} t_{k}^{j} \alpha_{k}(\lambda)=\frac{t_{0}^{j}}{\lambda^{j}}
$$

for some constant $t_{0}^{j}$. This gives us $K+1$ equations corresponding to $j=0,1, \cdots, K$. Since for each of these $K+1$ equations, we have the same set of $\left\{\alpha_{k}(\lambda)\right\}_{k=1}^{K}$ on the left-hand side, there must exist $p^{0}, p^{1}, \cdots, p^{K}$ not all equal to zero such that

$$
\sum_{j=0}^{K} p^{j} \sum_{k=1}^{K} t_{k}^{j} \alpha_{k}(\lambda)=0 .
$$

By (41), this implies

$$
\sum_{j=0}^{K} p^{j} \frac{t_{0}^{j}}{\lambda^{j}}=0 .
$$

Since $1, \frac{1}{\lambda}, \cdots, \frac{1}{\lambda^{K}}$ are linearly independent, their linear combination vanishes only if all coefficients are equal to zero, i.e., $p^{j} t_{0}^{j}=0$ for all $j$. Since $p^{j}$ are not all equal to zero, there must be some $j^{\prime}$ so that $t_{0}^{j^{\prime}}=0$. We will show that this cannot happen.

If $t_{0}^{j^{\prime}}=0$, by (41) we have

$$
\sum_{k=1}^{K} t_{k}^{j^{\prime}} \alpha_{k}(\lambda)=0
$$

Since $t_{1}^{j^{\prime}}, t_{2}^{j^{\prime}}, \cdots, t_{K}^{j^{\prime}}$ are not all equal to zero, take any $k^{\prime} \in\{1,2, \cdots, K\}$ such that $t_{k^{\prime}}^{j^{\prime}} \neq 0$, and we can rewrite $(42)$ as

$$
\alpha_{k^{\prime}}(\lambda)=-\sum_{k \neq k^{\prime}} \frac{t_{k}^{j^{\prime}}}{t_{k^{\prime}}^{j^{\prime}}} \alpha_{k}(\lambda) .
$$

Plugging this into (2) produces

$$
\begin{aligned}
I(\lambda \rho) & =\sum_{k \neq k^{\prime}} \alpha_{k}(\lambda) f_{k}(\rho)-\sum_{k \neq k^{\prime}} \frac{t_{k}^{j^{\prime}}}{t_{k^{\prime}}^{j^{\prime}}} \alpha_{k}(\lambda) f_{k^{\prime}}(\rho) \\
& =\sum_{k \neq k^{\prime}} \alpha_{k}(\lambda)\left[f_{k}(\rho)-\frac{t_{k}^{j^{\prime}}}{t_{k^{\prime}}^{j^{\prime}}} f_{k^{\prime}}(\rho)\right] .
\end{aligned}
$$


Thus, we have (no more than) $(K-1)$-fund separation, with the separating funds being $f_{k}(\rho)-\frac{t_{k}^{j^{\prime}}}{t_{k^{\prime}}^{j^{\prime}}} f_{k^{\prime}}(\rho)$ for $k \neq k^{\prime}$. This contradicts $K$-fund separation.

Hence, we must have $t_{0}^{j} \neq 0$ for all $j=0,1, \cdots, K$. This in turn implies that there exists some $j$ such that $M_{j}(\lambda)$ is invertible at some $\lambda>0$. Consequently (3) can be obtained as the necessary condition for $K$-fund separation.

((3) implies $K$-fund separation) To establish sufficiency, we need to show that any utility function satisfying (3) indeed exhibits $K$-fund separation. To this end, the optimal consumption porfolio can be written as

$$
\begin{aligned}
& I(\lambda \rho) \\
& =\sum_{k=1}^{J}(\lambda \rho)^{\gamma_{k}} P_{k, 1}(\log (\lambda \rho)) \cos \left(b_{k} \log (\lambda \rho)\right)+\sum_{k=1}^{J}(\lambda \rho)^{\gamma_{k}} P_{k, 2}(\log (\lambda \rho)) \sin \left(b_{k} \log (\lambda \rho)\right) \\
& =\sum_{k=1}^{J}(\lambda \rho)^{\gamma_{k}} \cos \left(b_{k} \log (\lambda \rho)\right) \sum_{j=0}^{d_{k, 1}} C_{k, 1, j}(\log (\lambda \rho))^{j}+\sum_{k=1}^{J}(\lambda \rho)^{\gamma_{k}} \sin \left(b_{k} \log (\lambda \rho)\right) \sum_{j=0}^{d_{k, 2}} C_{k, 2, j}(\log (\lambda \rho))^{j} \\
& =\sum_{k=1}^{J}(\lambda \rho)^{\gamma_{k}}\left[\begin{array}{c}
\cos \left(b_{k} \log \lambda\right) \cos \left(b_{k} \log \rho\right) \\
-\sin \left(b_{k} \log \lambda\right) \sin \left(b_{k} \log \rho\right)
\end{array}\right] \sum_{l=0}^{d_{k, 1}}(\log \rho)^{l} \sum_{j=l}^{d_{k, 1}} C_{k, 1, j}\left(\begin{array}{c}
j \\
l
\end{array}\right)(\log \lambda)^{j-l} \\
& +\sum_{k=1}^{J}(\lambda \rho)^{\gamma_{k}}\left[\begin{array}{c}
\sin \left(b_{k} \log \lambda\right) \cos \left(b_{k} \log \rho\right) \\
+\cos \left(b_{k} \log \lambda\right) \sin \left(b_{k} \log \rho\right)
\end{array}\right] \sum_{l=0}^{d_{k, 2}}(\log \rho)^{l} \sum_{j=l}^{d_{k, 2}} C_{k, 2, j}\left(\begin{array}{c}
j \\
l
\end{array}\right)(\log \lambda)^{j-l} \\
& =\sum_{\left\{k: b_{k}=0\right\}} \sum_{l=0}^{d_{k}} \alpha_{k, l}(\lambda) f_{k, l}(\rho)+\sum_{\left\{k: b_{k} \neq 0\right\}} \sum_{l=0}^{d_{k}}\left[\alpha_{k, 1, l}(\lambda) f_{k, 1, l}(\rho)+\alpha_{k, 2, l}(\lambda) f_{k, 2, l}(\rho)\right],
\end{aligned}
$$

where $f_{k, l}(\rho), f_{k, 1, l}(\rho), f_{k, 2, l}(\rho)$ and $\alpha_{k, l}(\lambda), \alpha_{k, 1, l}(\lambda), \alpha_{k, 2, l}(\lambda)$ are given by $(4)-$ (6) and (7)-(9). Since $f_{k, l}(\rho), f_{k, 1, l}(\rho)$ and $f_{k, 2, l}(\rho)$ are linearly independent, and $\alpha_{k, l}(\lambda), \alpha_{k, 1, l}(\lambda), \alpha_{k, 2, l}(\lambda)$ are also linearly independent, $K$-fund separation holds with $K=\sum_{k=1}^{J}\left(d_{k}+1\right)\left(1+\mathbf{1}_{b_{k} \neq 0}\right)$.

Proof of Proposition 1: Differentiating (34) yields

$$
I^{\prime}(\xi)=\sum_{k=1}^{K} \gamma_{k} C_{k} \xi^{\gamma_{k}-1} .
$$

Strict concavity implies $I^{\prime}(\xi) \leq 0$ for all $\xi>0$.

To show $\gamma_{1} C_{1}<0$, it is useful to rewrite (43) as

$$
I^{\prime}(\xi)=\xi^{\gamma_{1}-1} \sum_{k=1}^{K} \gamma_{k} C_{k} \xi^{\gamma_{k}-\gamma_{1}} .
$$


Since $\xi^{\gamma_{1}-1}>0$, strict concavity requires $\forall \xi>0$,

$$
\sum_{k=1}^{K} \gamma_{k} C_{k} \xi^{\gamma_{k}-\gamma_{1}} \leq 0
$$

Given $\gamma_{1}<\gamma_{2}<\cdots<\gamma_{K}$, we have that for all $k=2,3, \cdots, K$,

$$
\lim _{\xi \rightarrow 0} \xi^{\gamma_{k}-\gamma_{1}}=0
$$

This implies

$$
\lim _{\xi \rightarrow 0} \sum_{k=1}^{K} \gamma_{k} C_{k} \xi^{\gamma_{k}-\gamma_{1}}=\gamma_{1} C_{1} .
$$

Hence, to ensure $I^{\prime}(\xi) \leq 0$ when $\xi$ approaches zero, we must have $\gamma_{1} C_{1}<0$.

To show $\gamma_{K} C_{K}<0$, it is useful to rewrite (43) as

$$
I^{\prime}(\xi)=\xi^{\gamma_{K}-1} \sum_{k=1}^{K} \gamma_{k} C_{k} \xi^{\gamma_{k}-\gamma_{K}} .
$$

Since $\xi^{\gamma_{K}-1}>0$, strict concavity requires $\forall \xi>0$,

$$
\sum_{k=1}^{K} \gamma_{k} C_{k} \xi^{\gamma_{k}-\gamma_{K}} \leq 0 .
$$

Given $\gamma_{1}<\gamma_{2}<\cdots<\gamma_{K}$, we have that for all $k=1,2, \cdots, K-1$,

$$
\lim _{\xi \rightarrow+\infty} \xi^{\gamma_{k}-\gamma_{K}}=0
$$

This implies

$$
\lim _{\xi \rightarrow+\infty} \sum_{k=1}^{K} \gamma_{k} C_{k} \xi^{\gamma_{k}-\gamma_{K}}=\gamma_{K} C_{K} .
$$

Hence, to ensure $I^{\prime}(\xi) \leq 0$ when $\xi$ approaches infinity, we must have $\gamma_{K} C_{K}<0$.

Proof of Proposition 2: Our goal is to show that in each case we can find $\xi>0$ such that $I^{\prime}(\xi)>0$.

Case 1: When $P(\cdot)$ is of degree 0 , then $I(\xi)$ is a constant, which clearly cannot happen.

When $P(\cdot)$ is of an even degree $d \geq 2$, we can rewrite $I(\xi)$ as

$$
I(\xi)=\sum_{k=0}^{d} C_{k}(\log \xi)^{k},
$$


with $C_{d} \neq 0$. Differentiating $I$ yields

$$
\begin{aligned}
I^{\prime}(\xi) & =\sum_{k=1}^{d} k C_{k} \frac{(\log \xi)^{k-1}}{\xi} \\
& =\frac{(\log \xi)^{d-2}}{\xi} \sum_{k=1}^{d} k C_{k}(\log \xi)^{k-d+1}
\end{aligned}
$$

Since $d \geq 2$ is even, we have $\frac{(\log \xi)^{d-2}}{\xi}>0$ for all $\xi \neq 1$. Thus, the sign of $I^{\prime}(\xi)$ depends on that of $\sum_{k=1}^{d} k C_{k}(\log \xi)^{k-d+1}$. It can be verified that

$$
\lim _{\xi \rightarrow 0} \sum_{k=1}^{d} k C_{k}(\log \xi)^{k-d+1}=+\infty
$$

when $C_{d}<0$, and

$$
\lim _{\xi \rightarrow+\infty} \sum_{k=1}^{d} k C_{k}(\log \xi)^{k-d+1}=+\infty,
$$

when $C_{d}>0$. This implies that we can always find $\xi$ small or large enough such that $\sum_{k=1}^{d} k C_{k}(\log \xi)^{k-d+1}>0$ and hence $I^{\prime}(\xi)>0$, violating concavity.

Case 2: When $P(\cdot)$ is of an odd degree $d \geq 1$, we can rewrite $I(\xi)$ as

$$
I(\xi)=\sum_{k=0}^{d} C_{k} \xi^{\gamma}(\log \xi)^{k}
$$

with $\gamma \neq 0$ and $C_{d} \neq 0$. Differentiating $I$ yields

$$
\begin{aligned}
I^{\prime}(\xi) & =\xi^{\gamma-1}\left(\sum_{k=0}^{d-1}\left(C_{k} \gamma+C_{k+1}(k+1)\right)(\log \xi)^{k}+C_{d} \gamma(\log \xi)^{d}\right) \\
& =\xi^{\gamma-1}(\log \xi)^{d-1}\left(\sum_{k=0}^{d-1}\left(C_{k} \gamma+C_{k+1}(k+1)\right)(\log \xi)^{k-d+1}+C_{d} \gamma \log \xi\right) .
\end{aligned}
$$

Since $d \geq 1$ is odd, we have $\xi^{\gamma-1}(\log \xi)^{d-1}>0$ for all $\xi \neq 1$. Thus, the sign of $I^{\prime}(\xi)$ depends on that of $\sum_{k=0}^{d-1}\left(C_{k} \gamma+C_{k+1}(k+1)\right)(\log \xi)^{k-d+1}+C_{d} \gamma \log \xi$. It can be verified that

$$
\lim _{\xi \rightarrow 0} \sum_{k=0}^{d-1}\left(C_{k} \gamma+C_{k+1}(k+1)\right)(\log \xi)^{k-d+1}+C_{d} \gamma \log \xi=+\infty,
$$


when $C_{d} \gamma<0$, and

$$
\lim _{\xi \rightarrow+\infty} \sum_{k=0}^{d-1}\left(C_{k} \gamma+C_{k+1}(k+1)\right)(\log \xi)^{k-d+1}+C_{d} \gamma \log \xi=+\infty,
$$

when $C_{d} \gamma>0$. This implies that we can always find $\xi$ small or large enough such that $\sum_{k=0}^{d-1}\left(C_{k} \gamma+C_{k+1}(k+1)\right)(\log \xi)^{k-d+1}+C_{d} \gamma \log \xi>0$ and hence $I^{\prime}(\xi)>0$, violating concavity.

Case 3: In this case, we can rewrite $I(\xi)$ as

$$
I(\xi)=\sum_{k=1}^{J} \sum_{j=0}^{d_{k}}\left[C_{k, 1, j} \cos \left(b_{k} \log \xi\right)+C_{k, 2, j} \sin \left(b_{k} \log \xi\right)\right] \xi^{\gamma_{k}}(\log \xi)^{j}
$$

where $b_{k} \neq 0$, and at least one of $C_{k, 1, d_{k}}$ and $C_{k, 2, d_{k}}$ is non-zero for each $k$. Without loss of generality, assume $\gamma_{1}<\gamma_{2}<\cdots<\gamma_{J}$. Differentiating $I$ yields

$$
\begin{aligned}
I^{\prime}(\xi)= & \sum_{k=1}^{J} \xi^{\gamma_{k}-1} \\
& \left\{\begin{array}{c}
\sum_{j=0}^{d_{k}-1}\left[\begin{array}{c}
\left(C_{k, 1, j} \gamma_{k}+C_{k, 2, j} b_{k}+C_{k, 1, j+1}(j+1)\right) \cos \left(b_{k} \log \xi\right) \\
-\left(C_{k, 1, j} b_{k}-C_{k, 2, j} \gamma_{k}-C_{k, 2, j+1}(j+1)\right) \sin \left(b_{k} \log \xi\right)
\end{array}\right](\log \xi)^{j} \\
+\left[\begin{array}{c}
\left(C_{k, 1, d_{k}} \gamma_{k}+C_{k, 2, d_{k}} b_{k}\right) \cos \left(b_{k} \log \xi\right) \\
-\left(C_{k, 1, d_{k}} b_{k}-C_{k, 2, d_{k}} \gamma_{k}\right) \sin \left(b_{k} \log \xi\right)
\end{array}\right](\log \xi)^{d_{k}}
\end{array}\right\} \\
= & \xi^{\gamma_{J}-1} \sum_{k=1}^{J} \xi^{\gamma_{k}-\gamma_{J}(\log \xi)^{d_{k}}} \\
& \left\{\begin{array}{c}
\sum_{j=0}^{d_{k}-1}\left[\begin{array}{c}
\left(C_{k, 1, j} \gamma_{k}+C_{k, 2, j} b_{k}+C_{k, 1, j+1}(j+1)\right) \cos \left(b_{k} \log \xi\right) \\
-\left(C_{k, 1, j} b_{k}-C_{k, 2, j} \gamma_{k}-C_{k, 2, j+1}(j+1)\right) \sin \left(b_{k} \log \xi\right)
\end{array}\right](\log \xi)^{j-d_{k}} \\
+\left[\begin{array}{c}
\left(C_{k, 1, d_{k}} \gamma_{k}+C_{k, 2, d_{k}} b_{k}\right) \cos \left(b_{k} \log \xi\right) \\
-\left(C_{k, 1, d_{k}} b_{k}-C_{k, 2, d_{k}} \gamma_{k}\right) \sin \left(b_{k} \log \xi\right)
\end{array}\right]
\end{array}\right\} .
\end{aligned}
$$

It can be verified that

$$
\lim _{\xi \rightarrow+\infty} I^{\prime}(\xi)=\lim _{\xi \rightarrow+\infty} \xi^{\gamma_{J}-1}(\log \xi)^{d_{J}}\left[\begin{array}{c}
\left(C_{J, 1, d_{J}} \gamma_{J}+C_{J, 2, d_{J}} b_{J}\right) \cos \left(b_{J} \log \xi\right) \\
-\left(C_{J, 1, d_{J}} b_{J}-C_{J, 2, d_{J}} \gamma_{J}\right) \sin \left(b_{J} \log \xi\right)
\end{array}\right]
$$

which does not converge but instead switches between positive and negative values due to the cyclicality of the trigonometric terms. This implies that we can always find $\xi$ such that $I^{\prime}(\xi)>0$, violating concavity. 


\section{References}

[1] Bawa, Vijay S., and Eric B. Lindenberg, 1977. Capital market equilibrium in a mean-lower partial moment framework. Journal of Financial Economics 5, 189-200.

[2] Black, Fischer, 1972. Capital market equilibrium with restricted borrowing. Journal of Business 45, 444-455.

[3] Bôcher, Maxime, 1900. The theory of linear dependence. Annals of Mathematics $2,81-96$.

[4] Cass, David, and Joseph E. Stiglitz, 1970. The structure of investor preferences and asset returns, and separability in portfolio allocation: a contribution to the pure theory of mutual funds. Journal of Economic Theory 2, 122-160.

[5] Chen, An, Antoon Pelsser, and Michel Vellekoop, 2011. Modeling non-monotone risk aversion using SAHARA utility functions. Journal of Economic Theory 146, 2075-2092.

[6] Dybvig, Philip H., and Stephen A. Ross, 1982. Portfolio efficient sets. Econometrica 50, 1525-1546.

[7] Hakansson, Nils H., 1969. Risk disposition and the separation property in portfolio selection. Journal of Financial and Quantitative Analysis 4, 401-416.

[8] Hoene-Wronski, Józef, 1812. Réfutation de la théorie des fonctions analytiques de Lagrange. Paris.

[9] Kadan, Ohad, Fang Liu, and Suying Liu, 2016. Generalized systematic risk. American Economic Journal: Microeconomics, forthcoming.

[10] Machina, Mark J., 1982. "Expected utility" analysis without the independence axiom. Econometrica 50, 277-323.

[11] Muir, Thomas, 1882. A treatise on the theory of determinants. Macmillan.

[12] Pye, Gordon, 1967. Portfolio selection and security prices. Review of Economics and Statistics 49, 111-115. 
[13] Ross, Stephen A., 1978. Mutual fund separation in financial theory: the separating distributions. Journal of Economic Theory 17, 254-286.

[14] Samuelson, Paul A., 1967. General proof that diversification pays. Journal of Financial and Quantitative Analysis 2, 1-13.

[15] Tobin, James, 1958. Liquidity preference as behavior towards risk. Review of Economic Studies 25, 63-86. 